\title{
Consequentialism and Non-Consequentialism: The Axiomatic Approach*
}

\author{
Kotaro Suzumura \\ The Institute of Economic Research \\ Hitotsubashi University \\ Naka 2-1, Kunitachi, Tokyo 186-8603, Japan \\ Phone and Fax: + 81425808353 \\ E-mail: suzumura@ier.hit-u.ac.jp
}

YONGSHENG XU

Graduate School of Public Finance and Policy

Central University of Finance and Economics

Beijing, 100081, China

and

Department of Economics

Andrew Young School of Policy Studies

P.O. Box 3992, Georgia State University

Atlanta, GA 30302, U.S.A.

Phone: 404651 2769, Fax: 4046514985

E-mail: yxu3@gsu.edu

This Version: August 2006

* We are grateful to Kenneth Arrow, Walter Bossert, Wulf Gaertner, Prasanta Pattanaik and Amartya Sen, with whom we had several occasions to discuss the subject matter of this paper. Thanks are also due to Y. Iwata and T. Sakai for several suggestions while we were preparing this paper. Needless to say, they should not be held responsible for any defects that may still remain. Financial support through a Grant-in-Aid for Scientific Research from the Ministry of Education, Culture, Sports, Science and Technology of Japan is gratefully acknowledged. 


\begin{abstract}
Most, if not at all, practitioners of welfare economics and social choice theory are presumed to be welfaristic in their conviction. Indeed, they evaluate the goodness of an economic policy and/or economic system in terms of the welfare that people receive at the culmination outcomes thereby generated. Recent years have witnessed a substantial upsurge of interest in the non-welfaristic bases, or even the non-consequentialist bases, of welfare economics and social choice theory. Capitalizing on the axiomatic approach which we explored in the recent past, we try to provide a coherent analysis of consequentialism vis-à-vis non-consequentialism. To begin with, we develop an abstract framework in which the primitive of our analysis is a preference ordering held by an evaluator over the pairs of culmination outcomes and opportunity sets from which those culmination outcomes are chosen. As a partial test to see how much relevance can be claimed of the axiomatized concepts of consequentialism and non-consequentialism, two simple applications of this abstract framework are worked out. The first application is to the Arrovian social choice theory and the second application is to the analysis of ultimatum games.
\end{abstract}

JEL Classification Numbers: D00, D60, D63, D71 


\section{Introduction}

It is undeniable that most, if not all, welfare economists and social choice theorists are welfaristic in their conviction in the sense that they regard an economic policy and/or economic system to be satisfactory if and only if it is warranted to generate culmination outcomes which score high in the measuring rod of social welfare. It is equally undeniable that there do exist people who care not only about welfaristic features of the consequences, but also about non-welfaristic features of the consequences, or even non-consequential features such as the procedures through which these consequences are brought about, or the opportunity sets from which these consequences are chosen. ${ }^{1}$ Since welfare economics and social choice theory are concerned with the design and implementation of an economic policy or economic system from the viewpoint of persons constituting the society, even those welfare economists and social choice theorists with strong welfaristic conviction should be ready to take the judgements of people with non-welfaristic or non-consequentialist convictions into account in order not to be paternalistic in their social welfare analysis. The necessity to develop an analytical framework which enables us to examine the preferences of non-welfaristic and/or non-consequentialist people is all too clear. In a sequence of recent articles, Suzumura and $\mathrm{Xu}(2001 ; 2003 ; 2004)$ developed such an analytical framework and explored its implications in the context of Arrovian social choice theory.

The starting point of this analysis is to allow an individual to express his preferences of the following type: it is better for him that an outcome $x$ is realized from the opportunity set $A$ than that another outcome $y$ is realized from the opportunity set $B .^{2}$ Note, in particular, that he is expressing his intrinsic valuation of the opportunity for choice if he prefers choosing an outcome $x$ from an opportunity set $A$,

\footnotetext{
${ }^{1}$ The relevance of procedures or processes in decision-making has been argued by various writers such as Simon $(1976 ; 1978)$, Sen $(1995 ; 1996)$ and Suzumura $(1999 ; 2000)$, and it has been empirically observed in several settings in economics including experimental games (see, for example, Rabin (1993, 2002) and Bolton, Brandts and Ockenfels (2005) where it is found that players care not only about culmination outcomes, but also about the processes/procedures through which those culmination outcomes are brought about), and in empirical measurement of happiness (see, for example, Frey and Stutzer (2004) where they find that people care about culmination outcomes as well as procedures, especially participation rights in decision-making).

${ }^{2}$ Much attention has been focussed on the opportunity set evaluation, beginning with Sen (1985; 1988). See, among many others, Bossert, Pattanaik and Xu (1994), Gravel (1994; 1998), Jones and Sugden (1982), Pattanaik and Xu (1990; 1998), Sen (1993; 1996), and Suzumura (1999; 2000).
} 
where $\{x\}$ is a proper subset of $A$, rather than choosing the same outcome $x$ from the singleton opportunity set $\{x\}$. Using this analytical framework, we can put forward a concise definition of consequentialism and non-consequentialism, and characterize these concepts in terms of a few simple axioms.

The structure of this paper is as follows. In Section 2, we present the basic notations and definitions. Section 3 discusses the basic axioms which are assumed throughout this paper. Some simple implications of these axioms are also identified in this section. In Section 4, we define and characterize axiomatically the concept of extreme consequentialism and strong consequentialism. We then turn in Section 5 to the concepts of extreme non-consequentialism and strong non-consequentialism and their axiomatic characterizations. Observe that these axiomatizations of consequentialism and non-consequentialism are concerned only with rather extreme cases where unequivocal priority is given to eventual consequences (resp. opportunities) not only in the case of extreme consequentialism (resp. extreme non-consequentialism) but also in the case of strong consequentiaslim (resp. strong non-consequentialism). Section 6 introduces a more general framework so that active interactions between consequential considerations and procedural considerations are allowed to play an essential role. In other words, it develops a framework which allows trade-offs between the value of culmination outcomes and the richness of background opportunities. In Section 7, two simple applications of our analytical framework are briefly pursued. The first application is to the Arrovian social choice theory. Observe that Arrow's theoretical framework hinges squarely on the implicit assumption that all individuals in the society are consequentialist in nature. To gauge the extent to which Arrow's impossibility theorem and the resolution thereof hinge on this basic feature of his framework, two extended frameworks, in which individuals are supposed to express their preferences not only about culmination outcomes, but also about opportunity sets from which outcomes are chosen, are explored. The second application is to the ultimatum games. Capitalizing on the observations based on the experimental ultimatum games, we define and characterize extreme consequentialism, consequentialism, and fairness-conscious non-consequentialism in this context. Section 8 concludes this paper with several qualifications and observations. 


\section{Basic Notations and Definitions}

Let $X$ be the universal set which contains all mutually exclusive and jointly exhaustive social states. The elements of $X$ will be denoted by $x, y, z, \cdots$, and they are interpreted as culmination outcomes. $K$ denotes the set of all non-empty and finite subsets of $X$. The elements in $K$ will be denoted by $A, B, C, \cdots$, and they are interpreted as opportunity sets. Let $\Omega=\{(x, A): x \in A, A \in K\}$. Elements of $\Omega$ will be denoted by $(x, A),(y, B),(z, C), \cdots$, and they are called extended alternatives with the intended interpretation that the culmination outcome $x$ is chosen from the opportunity set $A$.

Let $\succeq$ be a reflexive, complete and transitive binary relation over $\Omega$. The asymmetric and symmetric parts of $\succeq$ will be denoted by $\succ$ and $\sim$, respectively. For any $(x, A),(y, B) \in \Omega,(x, A) \succeq(y, B)$ is interpreted as "choosing $x$ from the opportunity set $A$ is at least as good as choosing $y$ from the opportunity set $B$." The binary relation $\succeq$ may be called an extended preference ordering. In this extended framework, when the decision maker's extended preference is such that $(x, A) \succ(x,\{x\})$ for some $A \in \Omega$, it may be construed that he attaches intrinsic value as well as instrumental value to the opportunity set $A$. The following definitions are meant to capture the attitudes of the decision-making agent towards extended alternatives.

Definition 1. $\succeq$ is said to be

(1.1.) extremely consequential if, for all $(x, A),(x, B) \in \Omega,(x, A) \sim(x, B)$;

(1.2.) strongly consequential if, for all $x, y \in X$ and all $(x, A),(y, B) \in \Omega,(x,\{x\}) \sim$ $(y,\{y\})$ implies $[(x, A) \succeq(y, B) \Leftrightarrow|A| \geq|B|]$, and $(x,\{x\}) \succ(y,\{y\})$ implies $(x, A) \succ(y, B)$;

(1.3.) extremely non-consequential if, for all $(x, A),(y, B) \in \Omega,(x, A) \succeq(y, B) \Leftrightarrow$ $|A| \geq|B|$

(1.4.) strongly non-consequential if, for all $x, y \in X$ and all $(x, A),(y, B) \in \Omega,|A|>$ $|B| \Rightarrow(x, A) \succ(y, B)$, and $|A|=|B| \Rightarrow[(x,\{x\}) \succeq(y,\{y\}) \Leftrightarrow(x, A) \succeq(y, B)]$.

Thus, according to the extreme consequentialism, two extended alternatives $(x, A)$ and $(y, B)$ in $\Omega$ are judged to be indifferent as long as $x=y$, no matter how the opportunity sets $A$ and $B$ differ from each other. In other words, an extreme consequentialist cares only about culmination outcomes and pays no attention to the background 
opportunity set. The strong consequentialism, on the other hand, stipulates that, in evaluating two extended alternatives $(x, A)$ and $(y, B)$ in $\Omega$, the opportunity sets $A$ and $B$ do not matter when the decision-making agent has a strict extended preference for $(x,\{x\})$ against $(y,\{y\})$, and it is only when the decision-making agent is indifferent between $(x,\{x\})$ and $(y,\{y\})$ that the opportunity sets $A$ and $B$ matter in ranking $(x, A)$ vis-à-vis $(y, B)$.

The extreme non-consequentialism may be regarded as the polar extreme case of consequentialism in that, in evaluating two extended alternatives $(x, A)$ and $(y, B)$ in $\Omega$, the outcomes $x$ and $y$ are not valued at all and the richness of opportunities reflected by the opportunity sets $A$ and $B$ exhausts everything that matters. In its complete neglect of culmination outcomes, the extreme non-consequentialism is indeed extreme, but it captures the sense in which people may claim: "Give me liberty, or give me death." It is in the similar vein that, in evaluating two extended alternatives $(x, A)$ and $(y, B)$ in $\Omega$, the strong non-consequentialism ignores the culmination outcomes $x$ and $y$ when the two opportunity sets $A$ and $B$ have different cardinality. It is only when the two opportunity sets $A$ and $B$ have identical cardinality that the culmination outcomes $x$ and $y$ have something to say in ranking $(x, A)$ vis-à-vis $(y, B)$.

\section{Basic Axioms and Their Implications}

In this section, we introduce three basic axioms for the extended preference ordering $\succeq$, which are proposed in Suzumura and Xu (2001; 2003), and present their implications.

Independence (IND): For all $(x, A),(y, B) \in \Omega$, and all $z \in X \backslash A \cup B,(x, A) \succeq$ $(y, B) \Leftrightarrow(x, A \cup\{z\}) \succeq(y, B \cup\{z\})$.

Simple Indifference (SI): For all $x \in X$, and all $y, z \in X \backslash\{x\},(x,\{x, y\}) \sim$ $(x,\{x, z\})$.

Simple monotonicity (SM): For all $(x, A),(x, B) \in \Omega$, if $B \subseteq A$, then $(x, A) \succeq$ $(x, B)$.

The axiom (IND) can be regarded as the counterpart of an independence property used in the literature on ranking opportunity sets in terms of the freedom of 
choice; see, for example, Pattanaik and Xu (1990). It requires that, for all extended alternatives $(x, A)$ and $(y, B)$ in $\Omega$, if an alternative $z$ is not in both $A$ and $B$, then the extended preference ranking over $(x, A \cup\{z\})$ and $(y, B \cup\{z\})$ corresponds to that over $(x, A)$ and $(y, B)$, regardless of the nature of the added alternative $z \in X \backslash A \cup B$. This axiom may be criticized along several lines. For example, when the freedom of choice is viewed as offering the decision-making agent a certain degree of diversity, (IND) may be problematic. It may be the case that the added alternative $z$ is very similar to some existing alternatives in $A$, but is very dis-similar to all the alternatives in $B$. In such a case, the addition of $z$ to $A$ may not increase the degree of freedom already offered by $A$, while adding $z$ to $B$ may increase the degree of freedom offered by $B$ substantially (see Bossert, Pattanaik and Xu (2003), and Pattanaik and Xu (2000; 2006) for some formal analysis of diversity). As a consequence, the decision-making agent may rank $(y, B \cup\{z\})$ strictly above $(x, A \cup\{z\})$ even though he ranks $(x, A)$ at least as high as $(y, B)$. It may also be argued that the added alternative may have "epistemic value" in that it tells us something important about the nature of the choice situation which prompts a rejection of (IND). Consider the following example which is due to Sen (1996, p.753): "If invited to tea $(t)$ by an acquaintance you might accept the invitation rather than going home $(O)$, that is, pick $t$ from the choice over $\{t, O\}$, and yet turn the invitation down if the acquaintance, whom you do not know very well, offers you a wider menu of having either tea with him or some heroin and cocain $(h)$; that is, you may pick $O$, rejecting $t$, from the larger set $\{t, h, O\}$. The expansion of the menu offered by this acquaintance may tell you something about the kind of person he is, and this could affect your decision even to have tea with him." This constitutes a clear violation of (IND) when $A=B$.

The axiom (SI) requires that choosing $x$ from "simple" cases, each involving two alternatives, is regarded as indifferent to each other. It should be noted that (SI) is subject to similar criticisms as (IND).

Finally, the axiom (SM) is a monotonicity property requiring that choosing an alternative $x$ from the set $A$ cannot be worse than choosing the same alternative $x$ from the subset $B$ of $A$. Various counterparts of (SM) in the literature on ranking opportunity sets in terms of the freedom of choice have been proposed and studied (see, for example, Bossert, Pattanaik and Xu (1994), Gravel (1994; 1998), Pattanaik and $\mathrm{Xu}(1990 ; 2000))$. It basically reflects the conviction that the decision-making agent is not averse to richer opportunities. In some cases, as argued in Dworkin 
(1982), richer opportunities can be a liability rather than a virtue. In such cases, the decision-making agent may prefer choosing $x$ from a smaller set to choosing the same $x$ from a larger set.

The following results, Propositions 1, 2 and 3, summarize the implications of the above three axioms.

Proposition 1. (Suzumura and Xu (2001), Theorem 3.1) If $\succeq$ satisfies (IND) and (SI), then for all $(x, A),(x, B) \in \Omega,|A|=|B| \Rightarrow(x, A) \sim(x, B)$.

Proof. Let $\succeq$ satisfy (IND) and (SI). Let $(x, A),(x, B) \in \Omega$ be such that $|A|=|B|$.

If $|A|=|B|=1$, then $A=B=\{x\}$. By reflexivity of $\succeq,(x, A) \sim(x, B)$ follows immediately. If $|A|=|B|=2$, then $(x, A) \sim(x, B)$ follows from (SI) directly. Consider now that $|A|=|B|=m+1$, where $\infty>m \geq 2$.

Suppose first that $A \cap B=\{x\}$. Let $A=\left\{x, a_{1}, \ldots, a_{m}\right\}$ and $B=\left\{x, b_{1}, \ldots, b_{m}\right\}$. From (SI), we must have $\left(x,\left\{x, a_{i}\right\}\right) \sim\left(x,\left\{x, b_{j}\right\}\right)$ for all $i, j=1, \ldots, m$. By (IND), from $\left(x,\left\{x, a_{2}\right\}\right) \sim\left(x,\left\{x, b_{1}\right\}\right)$, we obtain $\left(x,\left\{x, a_{1}, a_{2}\right\}\right) \sim\left(x,\left\{x, a_{1}, b_{1}\right\}\right)$, and from $\left(x,\left\{x, a_{1}\right\}\right) \sim\left(x,\left\{x, b_{2}\right\}\right)$, we obtain $\left(x,\left\{x, a_{1}, b_{1}\right\}\right) \sim\left(x,\left\{x, b_{1}, b_{2}\right\}\right)$. By the transitivity of $\succeq$, it follows that $\left(x,\left\{x, a_{1}, a_{2}\right\}\right) \sim\left(x,\left\{x, b_{1}, b_{2}\right\}\right)$. By using arguments similar to those above, from (IND) and by the transitivity of $\succeq$, we can obtain $(x, A) \sim(x, B)$.

Next, suppose that $A \cap B=\{x\} \cup C$ where $C \neq \emptyset$. When $A \backslash C=B \backslash C=\emptyset$, it must be the case that $A=B$. By reflexivity of $\succeq,(x, A) \sim(x, B)$ follows easily. Suppose therefore that $A \backslash C \neq \emptyset$. Note that $B \backslash C \neq \emptyset$ and $|A \backslash C|=|B \backslash C|$. From above, we must then have $(x,(A \backslash C) \cup\{x\}) \sim(x,(B \backslash C) \cup\{x\})$. By the repeated use of (IND), $(x, A) \sim(x, B)$ can be obtained.

Proposition 2. If $\succeq$ satisfies (IND) and (SI), then

(2.1) For all $x \in X$, if there exists $y \in X \backslash\{x\}$ such that $(x,\{x, y\}) \succ(x,\{x\})$, then for all $(x, A),(x, B) \in \Omega,|A| \geq|B| \Leftrightarrow(x, A) \succeq(x, B)$;

(2.2) For all $x \in X$, if there exists $y \in X \backslash\{x\}$ such that $(x,\{x, y\}) \sim(x,\{x\})$, then for all $(x, A),(x, B) \in \Omega,(x, A) \sim(x, B)$;

(2.3) For all $x \in X$, if there exists $y \in X \backslash\{x\}$ such that $(x,\{x\}) \succ(x,\{x, y\})$, then for all $(x, A),(x, B) \in \Omega,|A| \leq|B| \Leftrightarrow(x, A) \succeq(x, B)$.

Proof. Let $\succeq$ satisfy (IND) and (SI). We will give a proof for the case (2.1). The proofs for the cases (2.2) and (2.3) are similar and we omit them. Let $(x, A),(x, B) \in$ 
$\Omega$. If $|A|=|B|$, then, by Proposition $1,(x, A) \sim(x, B)$. Suppose now that $|A| \neq|B|$. Without loss of generality, let $|A|>|B|$. Consider $G \subset A$ such that $|G|=|B|$ and $x \in$ $G$. Then, by Proposition $1,(x, G) \sim(x, B)$. Let $A=G \cup H$ where $H=\left\{h_{1}, \cdots, h_{t}\right\}$. Let $G=\left\{x, g_{1}, \cdots, g_{r}\right\}$. Note that if there exists $y \in X \backslash\{x\}$ such that $(x,\{x, y\}) \succ$ $(x,\{x\})$, then, from Proposition 1 and by the transitivity of $\succeq$, it must be true that $(x,\{x, z\}) \succ(x,\{x\})$ for all $z \in X \backslash\{x\}$. In particular, $\left(x,\left\{x, h_{1}\right\}\right) \succ(x,\{x\})$. Therefore, by the repeated use of (IND), we have $\left(x, G \cup\left\{h_{1}\right\}\right) \succ(x, G)$. Similarly, $\left(x, G \cup\left\{h_{1}, h_{2}\right\}\right) \succ\left(x, G \cup\left\{h_{1}\right\}\right)$, and $\left(x, G \cup\left\{h_{1}, h_{2}, h_{3}\right\}\right) \succ\left(x, G \cup\left\{h_{1}, h_{2}\right\}\right)$, and $\cdots$, and $(x, G \cup H) \succ\left(x, G \cup H \backslash\left\{h_{t}\right\}\right)$. By the transitivity of $\succeq$, it follows that $(x, A) \succ(x, G)$. Then, noting that $|G|=|B|$, from Proposition 1 and the transitivity of $\succeq$, we have $(x, A) \succ(x, B)$.

Proposition 3. (Suzumura and Xu (2003), Lemma 3.1) Let $\succeq$ be an ordering over $\Omega$ satisfying (IND), (SI) and (SM). Then, for all $(a, A),(b, B) \in \Omega$, and all $x \in X \backslash A, y \in$ $X \backslash B,(a, A) \succeq(b, B) \Leftrightarrow(a, A \cup\{x\}) \succeq(b, B \cup\{y\})$.

Proof. Let $\succeq$ satisfy (IND), (SI) and (SM). Let $(a, A),(b, B) \in \Omega, x \in X \backslash A, y \in X \backslash B$ and $(a, A) \succeq(b, B)$. Because $\succeq$ is an ordering, we have only to show that $(a, A) \sim$ $(b, B) \Rightarrow(a, A \cup\{x\}) \sim(b, B \cup\{y\})$ and $(a, A) \succ(b, B) \Rightarrow(a, A \cup\{x\}) \succ(b, B \cup\{y\})$.

First, we show that

$$
(a, A) \sim(b, B) \Rightarrow(a, A \cup\{x\}) \sim(b, B \cup\{y\}) .
$$

Since $x \in X \backslash A$ and $y \in X \backslash B$, it is clear that $A \neq X$ and $B \neq X$. We consider three sub-cases: (i) $A=\{a\}$; (ii) $B=\{b\}$; and (iii) $|A|>1$ and $|B|>1$.

(i) $A=\{a\}$. In this case, we distinguish two sub-cases: (i.1) $x \notin B$; and (i.2) $x \in B$. Consider (i.1). Since $x \notin B$, it follows from $(a,\{a\}) \sim(b, B)$ and (IND) that $(a,\{a, x\}) \sim(b, B \cup\{x\})$. By Proposition 1, $(b, B \cup\{x\}) \sim(b, B \cup\{y\})$. Transitivity of $\succeq$ then implies that $(a,\{a, x\}) \sim(b, B \cup\{y\})$. Since $(a,\{a, x\})=(a, A \cup\{x\})$, we obtain (*) in this sub-case. Consider now (i.2), where $x \in B$. To begin with, consider the sub-case where $B \cup\{y\}=\{a, b\}$. Given that $x \in X \backslash A$ and $y \in X \backslash B$, we have $x=$ $b$ and $y=a$, hence $B=\{b\}$. Since $|X| \geq 3$, there exists $c \in X$ such that $c \notin\{a, b\}$. It follows from $(a,\{a\}) \sim(b,\{b\})=(b, B)$ and (IND) that $(a,\{a, c\}) \sim(b,\{b, c\})$. From Proposition 1, $(a,\{a, b\}) \sim(a,\{a, c\})$, and $(b,\{b, c\}) \sim(b,\{a, b\})$. Then, transitivity of $\succeq$ implies $(a,\{a, b\}) \sim(b,\{a, b\})$; that is, $(a,\{a, x\}) \sim(b, B \cup\{y\})$. Turn now to the sub-case where $B \cup\{y\} \neq\{a, b\}$. If $y \neq a$, starting with $(a,\{a\}) \sim(b, B)$, by $(\mathrm{IND}),(a,\{a, y\}) \sim(b, B \cup\{y\})$. By Proposition 1, $(a,\{a, x\}) \sim(a,\{a, y\})$. 
Transitivity of $\succeq$ implies that $(a,\{a, x\}) \sim(b, B \cup\{y\})$. If $y=a$, given that $|X| \geq 3$ and $B \cup\{y\} \neq\{a, b\}$, there exists $z \in B$ such that $z \notin\{a, b\}$. By Proposition $1,(b, B) \sim(b,(B \cup\{y\}) \backslash\{z\})$. From $(a,\{a\}) \sim(b, B)$, transitivity of $\succeq$ implies $(a,\{a\}) \sim(b,(B \cup\{y\}) \backslash\{z\})$. Now, noting that $z \neq a$, by (IND), $(a,\{a, z\}) \sim$ $(b, B \cup\{y\})$ holds. From Proposition $1,(a,\{a, x\}) \sim(a,\{a, z\})$. Transitivity of $\succeq$ now implies $(a,\{a, x\}) \sim(b, B \cup\{y\})$, which establishes $\left(^{*}\right)$ in this sub-case.

(ii) $B=\{b\}$. This case can be treated similarly as the case (i).

(iii) $|A|>1$ and $|B|>1$. Consider $A^{\prime},\left|A^{\prime \prime}\right| \in K$ such that $\{a, b\} \subset A^{\prime \prime} \subset$ $A^{\prime},\left|A^{\prime \prime}\right|=\min \{|A|,|B|\}>1,\left|A^{\prime}\right|=\max \{|A|,|B|\}>1$. Since $A \neq X$ and $B \neq X$, the existence of such $A^{\prime}$ and $A^{\prime \prime}$ is guaranteed. It should be clear that there exists $z \in X$ such that $z \notin A^{\prime}$. If $|A| \geq|B|$, consider $\left(a, A^{\prime}\right)$ and $\left(b, A^{\prime \prime}\right)$. From Proposition $1,\left(a, A^{\prime}\right) \sim\left(b, A^{\prime \prime}\right)$ follows from the construction of $A^{\prime}$ and $A^{\prime \prime}$, the assumption that $(a, A) \sim(b, B)$, and transitivity of $\succeq$. Note that there exists $z \in X \backslash A^{\prime}$. By (IND), $\left(a, A^{\prime} \cup\{z\}\right) \sim\left(b, A^{\prime \prime} \cup\{z\}\right)$. By virtue of Proposition 1, noting that $|A \cup\{x\}|=$ $\left|A^{\prime} \cup\{z\}\right|$ and $|B \cup\{y\}|=\left|A^{\prime \prime} \cup\{z\}\right|,(a, A \cup\{x\}) \sim(b, B \cup\{y\})$ follows easily from transitivity of $\succeq$. If $|A|<|B|$, consider $\left(a, A^{\prime \prime}\right)$ and $\left(b, A^{\prime}\right)$. Following a similar argument as above, we can show that $(a, A \cup\{x\}) \sim(b, B \cup\{y\})$. Thus, $\left(^{*}\right)$ is proved. The next order of our business is to show that

$$
(a, A) \succ(b, B) \Rightarrow(a, A \cup\{x\}) \succ(b, B \cup\{y\}) .
$$

As in the proof of $\left(^{*}\right)$, we distinguish three cases: (a) $A=\{a\}$; (b) $B=\{b\}$; and (c) $|A|>1$ and $|B|>1$.

(a) $A=\{a\}$. (a.1) $x \notin B$. In this sub-case, from $(a,\{a\}) \succ(b, B)$, by (IND), we obtain $(a,\{a, x\}) \succ(b, B \cup\{x\})$. By Proposition $1,(b, B \cup\{x\}) \sim(b, B \cup\{y\})$. Transitivity of $\succeq$ implies that $(a,\{a, x\})=(a, A \cup\{x\}) \succ(b, B \cup\{y\})$. (a.2) $x \in B$. If $B \cup\{y\}=\{a, b\}$, then, given that $x \notin A$ and $y \notin B$, we have $x=b$ and $y=a$. Since $|X| \geq 3$, there exists $c \in X$ such that $c \notin\{a, b\}$. It follows from $(a,\{a\}) \succ(b,\{b\})=$ $(b, B)$ and (IND) that $(a,\{a, c\}) \succ(b,\{b, c\})$. From Proposition 1, $(a,\{a, b\}) \sim$ $(a,\{a, c\})$ and $(b,\{b, c\}) \sim(b,\{a, b\})$. Transitivity of $\succeq \operatorname{implies}(a,\{a, b\}) \succ(b,\{a, b\})$, viz., $(a,\{a, x\})=(a, A \cup\{x\}) \succ(b, B \cup\{y\})$. If $B \cup\{y\} \neq\{a, b\}$, we consider (a.2.i) $y \neq a$ and (a.2.ii) $y=a$. Suppose that (a.2.i) $y \neq a$. From $(a,\{a\}) \succ(b, B)$, by $(\mathrm{IND}),(a,\{a, y\}) \succ(b, B \cup\{y\})$. By Proposition 1, $(a,\{a, x\}) \sim(a,\{a, y\})$. Transitivity of $\succeq$ now implies $(a,\{a, x\})=(a, A \cup\{x\}) \succ(b, B \cup\{y\})$. Suppose next that (a.2.ii) $y=a$. Since $|X| \geq 3$ and $B \cup\{y\} \neq\{a, b\}$, there exists $c \in B$ such that 
$c \notin\{a, b\}$. By Proposition 1, $(b, B) \sim(b,(B \cup\{y\}) \backslash\{c\})$. From $(a,\{a\}) \succ(b, B)$, by transitivity of $\succeq,(a,\{a\}) \succ(b,(B \cup\{y\}) \backslash\{c\})$. Now, noting that $c \neq a$, by (IND), $(a,\{a, c\}) \succ(b, B \cup\{y\})$. From Proposition 1, $(a,\{a, c\}) \sim(a,\{a, x\})$. Transitivity of $\succeq$ implies $(a,\{a, x\})=(a, A \cup\{x\}) \succ(b, B \cup\{y\})$.

(b) $B=\{b\}$. If $x \notin B$, it follows from $(a, A) \succ(b, B)$ and (IND) that ( $a, A \cup$ $\{x\}) \succ(b,\{b, x\})$. By Proposition $1,(b,\{b, x\}) \sim(b,\{b, y\})=(b, B \cup\{y\})$. Transitivity of $\succeq$ now implies $(a, A \cup\{x\}) \succ(b,\{b, y\})=(b, B \cup\{y\})$. If $x \in B$, then $x=b$. Consider first the case where $y=a$. If $A=\{a\}$, it follows from (a) that $(a,\{a, x\})=(a, A \cup\{x\}) \succ(b,\{b, y\})=(b, B \cup\{y\})$. Suppose $A \neq\{a\}$. Given that $x=b, y=a, x \notin A$, and $y \notin B$, and noting that $|X| \geq 3$, there exists $c \in A \backslash\{a, b\}$. From Proposition 1, $(a,(A \cup\{x\}) \backslash\{c\}) \sim(a, A)$. From transitivity of $\succeq$ and noting that $(a, A) \succ(b,\{b\}),(a,(A \cup\{x\}) \backslash\{c\}) \succ(b,\{b\})$ holds. By (IND), $(a, A \cup\{x\}) \succ(b,\{b, c\})$. From Proposition 1, $(b,\{b, y\}) \sim(b,\{b, c\})$. Therefore, $(a, A \cup\{x\}) \succ(b,\{b, y\})=(b, B \cup\{y\})$ follows easily from transitivity of $\succeq$. Consider next that $y \neq a$. If $y \notin A$, then, by (IND) and $(a, A) \succ(b,\{b\})$, we obtain $(a, A \cup\{y\}) \succ(b,\{b, y\})$ immediately. By Proposition 1, $(a, A \cup\{y\}) \sim(a, A \cup\{x\})$. Transitivity of $\succeq$ implies $(a, A \cup\{x\}) \succ(b,\{b, y\})=(b, B \cup\{y\})$. If $y \in A$, noting that $y \neq a, y \notin B$, and $x=b$, we have $|(A \cup\{x\}) \backslash\{y\}|=|A|$. By Proposition 1, $(a, A) \sim(a,(A \cup\{x\}) \backslash\{y\})$. Transitivity of $\succeq$ implies $(a,(A \cup\{x\}) \backslash\{y\}) \succ(b,\{b\})$. By (IND), it then follows that $(a, A \cup\{x\}) \succ(b,\{b, y\})=(b, B \cup\{y\})$.

(c) $|A|>1$ and $|B|>1$. This case is similar to the case (iii) above, and we may safely omit it.

Thus, $\left({ }^{* *}\right)$ is proved. $(*)$ together with $\left({ }^{* *}\right)$ completes the proof of Proposition 3.

\section{Consequentialism}

In this section, we present axiomatic characterizations of extreme consequentialism and strong consequentialism. To characterize these two versions of consequentialism, we consider the following two axioms, which are proposed in Suzumura and Xu (2001).

Local Indifference (LI): For all $x \in X$, there exists $(x, A) \in \Omega \backslash\{(x,\{x\})\}$ such that $(x,\{x\}) \sim(x, A)$. 
Local Strict Monotonicity (LSM): For all $x \in X$, there exists $(x, A) \in \Omega \backslash$ $\{(x,\{x\})\}$ such that $(x, A) \succ(x,\{x\})$.

Robustness (ROB): For all $x, y, z \in X$, all $(x, A),(y, B) \in \Omega$, if $(x,\{x\}) \succ(y,\{y\})$ and $(x, A) \succ(y, B)$, then $(x, A) \succ(y, B \cup\{z\})$.

The axiom (LI) is a mild requirement of extreme consequentialism: for each $x \in X$, there exists an opportunity set $A$ in $K$, which is distinct from $\{x\}$, such that choosing the alternative $x$ from $A$ is regarded as indifferent to choosing $x$ from the singleton set $\{x\}$. It may be regarded as a local property of extreme consequentialism. The axiom (LSM), on the other hand, requires that, for each $x \in X$, there exists an opportunity set $A$, which is distinct from $\{x\}$, such that choosing $x$ from the opportunity set $A$ is valued strictly higher than choosing $x$ from the singleton opportunity set $\{x\}$. It reflects the decision maker's desire to value opportunities at least in this very limited sense. The axiom (ROB) requires that, for all $x, y, z \in X$, all $(x, A),(y, B) \in \Omega$, if the decision maker values $(x,\{x\})$ higher than $(y,\{y\})$, and $(x, A)$ higher than $(y, B)$, then the addition of $z$ to $B$ while maintaining $y$ being chosen from $B \cup\{z\}$ will not affect the decision-making agent's value-ranking: $(x, A)$ is still valued higher than $(y, B)$.

Theorem 1. (Suzumura and Xu (2001), Theorem 4.1) $\succeq$ satisfies (IND), (SI) and (LI) if and only if it is extremely consequential.

Proof. It can be easily shown that, if $\succeq$ is extremely consequential, then it satisfies (IND), (SI) and (LI). Therefore, we have only to prove that, if $\succeq$ satisfies (IND), (SI) and $(\mathrm{LI})$, then, for all $(x, A),(x, B) \in \Omega,(x, A) \sim(x, B)$ holds.

Let $\succeq$ satisfy (IND), (SI) and (LI). First, observe that from Proposition 1, we have the following:

(T1.1) For all $(x, A),(x, B) \in \Omega,|A|=|B| \Rightarrow(x, A) \sim(x, B)$.

Thus, we have only to show that

(T1.2) For all $(x, A),(x, B) \in \Omega,|A|>|B| \Rightarrow(x, A) \sim(x, B)$.

From Proposition 2, and by (LI) and the completeness of $\succeq$, it must be true that (T1.3) For all distinct $x, y \in X,(x,\{x, y\}) \sim(x,\{x\})$. 
From (T1.3), by the repeated use of (IND), (T1.1) and the transitivity of $\succeq$, (T1.2) can be established.

Theorem 2. (Suzumura and Xu (2001), Theorem 4.2) $\succeq$ satisfies (IND), (SI), (LSM) and (ROB) if and only if it is strongly consequential.

Proof. Again, it can be shown that, if $\succeq$ is strongly consequential, then it satisfies (IND), (SI), (LSM) and (ROB). Therefore, we have only to prove that, if $\succeq$ satisfies (IND), (SI), (LSM) and (ROB), then, for all $(x, A),(y, B) \in \Omega,(x,\{x\}) \sim(y,\{y\})$ implies $[(x, A) \succeq(y, B) \Leftrightarrow|A| \geq|B|]$, and $(x,\{x\}) \succ(y,\{y\})$ implies $(x, A) \succ(y, B)$.

Let $\succeq$ satisfy (IND), (SI), (LSM) and (ROB). Note that, from Proposition 1, we have the following:

(T2.1) For all $x \in X$ and all $(x, A),(x, B) \in \Omega,|A|=|B| \Rightarrow(x, A) \sim(x, B)$.

Next, from Proposition 2, and by (LSM) and the completeness of $\succeq$, it must be true that

(T2.2) For all distinct $x, y \in X,(x,\{x, y\}) \succ(x,\{x\})$.

From (T2.2) and by the repeated use of (IND), we can derive the following:

(T2.3) For all $x \in X$ and all $(x, A),(x, B) \in \Omega,|A|>|B| \Rightarrow(x, A) \succ(x, B)$.

Now, for all $x, y \in X$, consider $(x,\{x\})$ and $(y,\{y\})$. If $(x,\{x\}) \sim(y,\{y\})$, then, since $X$ contains at least three alternatives, by IND, for all $z \in X \backslash\{x, y\}$, we must have $(x,\{x, z\}) \sim(y,\{y, z\})$. From (T2.1) and by the transitivity of $\succeq$, we then have $(x,\{x, y\}) \sim(y,\{x, y\})$. Then, by IND, we have $(x,\{x, y, z\}) \sim(y,\{x, y, z\})$. Since the opportunity sets are finite, by the repeated application of (T2.1) and (T2.3), the transitivity of $\succeq$ and (IND), we then obtain

(T2.4) For all $x, y \in X$ and all $(x, A),(y, B) \in \Omega$, if $(x,\{x\}) \sim(y,\{y\})$, then $|A| \geq$ $|B| \Leftrightarrow(x, A) \succeq(y, B)$.

If, on the other hand, $(x,\{x\}) \succ(y,\{y\})$, then, for all $z \in X$, (ROB) implies $(x,\{x\}) \succ(y,\{y, z\})$. Since opportunity sets are finite, by repeated use of (ROB), we then obtain $(x,\{x\}) \succ(y, A)$ for all $(y, A) \in \Omega$. Therefore, from (T2.1) and (T2.3), and by the transitivity of $\succeq$, we obtain 
(T2.5) For all $x, y \in X$ and all $(x, A),(y, B) \in \Omega$, if $(x,\{x\}) \succ(y,\{y\})$, then $(x, A) \succ$ $(y, B)$.

(T2.5), together with (T2.1), (T2.3) and (T2.4), completes the proof.

To conclude this section, we show that the characterization theorems we obtained, viz., Theorem 1 for extreme consequentialism and Theorem 2 for strong consequentialism, do not contain any redundancy by proving the independence of the axioms appearing in these theorems.

Proposition 4. The axioms (IND), (SI) and (LI) are independent.

Proof. We construct three examples to prove the proposition. In these examples, the respective axiom that is violated is indicated. Let $X=\{x, y, z\}$.

$(\mathrm{P} 4.1) \neg$ (IND): Consider $\succeq_{1}$ defined by $(x, X) \sim_{1}(y, X) \sim_{1}(z, X) \sim_{1}(x,\{x\}) \sim_{1}$ $(y,\{y\}) \sim_{1}(z,\{z\}) \succ_{1}(x,\{x, y\}) \sim_{1}(x,\{x, z\}) \sim_{1}(y,\{x, y\}) \sim_{1}(y,\{y, z\}) \sim_{1}$ $(z,\{x, z\}) \sim_{1}(z,\{y, z\}) . \succeq_{1}$ satisfies $(\mathrm{SI})$ and (LI), but it violates (IND) since $(x,\{x, y, z\}) \succ_{1}(x,\{x, z\})$ and $(x,\{x\}) \succ_{1}(x,\{x, y\})$.

$(\mathrm{P} 4.2) \neg(\mathrm{SI}):$ Consider $\succeq_{2}$ defined by $(x, X) \sim_{2}(y, X) \sim_{2}(z, X) \sim_{2}(x,\{x, y\}) \sim_{2}$ $(y,\{x, y\}) \sim_{2}(y,\{y, z\}) \sim_{2}(y,\{y\}) \sim_{2}(z,\{y, z\}) \succ_{2}(x,\{x, z\}) \sim_{2}(x,\{x\}) \sim_{2}$ $(z,\{x, z\}) \sim_{2}(z,\{z\}) . \succeq_{2}$ satisfies (IND) and (LI), but it violates (SI) since $(x,\{x, y\})$ $\succ_{2}(x,\{x, z\})$.

(P4.3) $\neg(\mathrm{LI})$ : Define $\succeq_{3}$ as a strong consequentialist's extended preference ordering. Then, $\succeq_{3}$ satisfies IND and SI, but it violates (LI) since $(x, X) \succ_{3}(x, A)$ for all $(x, A) \in \Omega \backslash\{(x, X)\}$.

Proposition 5. The axioms (IND), (SI), (LSM) and (ROB) are independent.

Proof. We construct four examples to prove the proposition. In these examples, the respective axiom that is violated is indicated. Let $X=\{x, y, z\}$.

(P5.1) $\neg(\mathrm{IND}): \succeq=\succeq_{1}$ defined in (P4.1) will suffice.

$(\mathrm{P} 5.2) \neg(\mathrm{SI}):$ Define $\succeq_{4}$ by $(x, X) \sim_{4}(y, X) \sim_{4}(z, X) \succ_{4}(x,\{x, y\}) \sim_{4}(y,\{x, y\})$ $\sim_{4}(z,\{x, z\}) \sim_{4}(z,\{y, z\}) \succ_{4}(x,\{x, z\}) \sim_{4}(y,\{y, z\}) \succ_{4}(x,\{x\}) \sim_{4}(y,\{y\}) \sim_{4}$ $(z,\{z\}) . \succeq_{4}$ satisfies (IND), (LSM) and (ROB), but it violates (SI) since $(x,\{x, y\}) \succ_{4}$ $(x,\{x, z\})$.

$(\mathrm{P} 5.3) \neg(\mathrm{LSM})$ : For all $(x, A),(y, B) \in \Omega$, define $\succeq_{5}$ by $(x, A) \sim_{5}(y, B)$. Clearly, $\succeq_{5}$ satisfies (IND), (SI) and (ROB), but it violates (LSM). 
$(\mathrm{P} 5.4) \neg$ (ROB): Define $\succeq_{6}$ by $(x, X) \sim_{6}(y, X) \sim_{6}(z, X) \succ_{6}(x,\{x, y\}) \sim_{6}$ $(x,\{x, z\}) \succ_{6}(y,\{x, y\}) \sim_{6}(y,\{y, z\}) \sim_{6}(z,\{z, y\}) \sim_{6}(z,\{z, x\}) \succ_{6}(x,\{x\}) \succ_{6}$ $(y,\{y\}) \sim_{6}(z,\{z\}) . \succeq_{6}$ satisfies (IND), (SI) and (LSM), but it violates (ROB) since $(x,\{x, y\}) \succ_{6}(y,\{x, y\})$ and $(y,\{x, y, z\}) \succ_{6}(x,\{x, y\})$.

\section{Non-Consequentialism}

To give characterizations of the extreme non-consequentialism and strong consequentialism, the following axioms will be used.

Indifference of No-Choice Situations (INS): For all $x, y \in X,(x,\{x\}) \sim$ $(y,\{y\})$.

Simple Preference for Opportunities (SPO): For all distinct $x, y \in X,(x,\{x, y\})$ $\succ\{y,\{y\})$.

The axiom (INS) requires that, in facing two choice situations in which each choice situation is restricted to a choice from a singleton set, the decision-making agent is indifferent between them. It thus conveys the idea that, in these simple cases, the decision-making agent feels that there is no real freedom of choice in each choice situation, so that he is ready to express his indifference between these simple choice situations regardless of the nature of the culmination outcomes. In a sense, it is the lack of freedom of choice that "forces" the decision-making agent to be indifferent between these situations. The underlying idea of (INS) is therefore similar to an axiom proposed by Pattanaik and Xu (1990) for ranking opportunity sets in terms of the freedom of choice, which requires that all singleton sets offer the decision-making agent the same amount of freedom of choice. The axiom (SPO) stipulates that it is always better for the agent to choose an outcome from the set containing two elements (one of which being the chosen culmination outcome) than to choose a culmination outcome from the singleton set. (SPO) therefore displays the decision-making agent's desire for having some genuine opportunities for choice. In this sense, (SPO) is in the same spirit as (LSM). However, as the following result shows, (SPO) is a stronger requirement than (LSM) in the presence of (IND) and (SI). 
Proposition 6. Suppose $\succeq$ satisfies (IND) and (SI). Then (SPO) implies (LSM).

Proof. Let $\succeq$ satisfy (IND), (SI) and (SPO). Let $x \in X$. For all $y \in X \backslash\{x\}$, by $(\mathrm{SPO}),(x,\{x, y\}) \succ(y,\{y\})$. Then, (IND) implies $(x,\{x, y, z\}) \succ(y,\{y, z\})$ for all $z \in X \backslash\{x, y\}$. By (SI), $(y,\{y, z\}) \sim(y,\{x, y\})$. It follows from the transitivity of $\succeq$ that $(x,\{x, y, z\}) \succ(y,\{x, y\})$. By (SPO), $(y,\{x, y\}) \succ(x,\{x\})$. Then, $(x,\{x, y, z\}) \succ(x,\{x\})$ follows from the transitivity of $\succeq$. Therefore, for $A=\{x, y, z\},(x, A) \succ(x,\{x\})$ holds. Thus, $\succeq$ satisfies (LSM).

Theorem 3. $\succeq$ satisfies (IND), (SI), (LSM) and (INS) if and only if it is extremely non-consequential.

Proof. It can be checked that if $\succeq$ is extremely non-consequential, then it satisfies (IND), (SI), (LSM) and (INS). Therefore, we have only to prove that, if $\succeq$ satisfies (IND), (SI), (LSM) and (INS), then, for all $(x, A),(y, B) \in \Omega,|A| \geq|B| \Leftrightarrow(x, A) \succeq$ $(y, B)$.

Let $\succeq$ satisfy (IND), (SI), (LSM) and (INS). First, we note that, following the similar proof method used for proving (T2.3), the following can be established:

(T3.1) For all $(x, A),(x, B) \in \Omega,|A|>|B| \Rightarrow(x, A) \succ(x, B)$.

Together with Proposition 1 and recollecting that $\succeq$ is complete, we must have the following:

(T3.2) For all $(x, A),(x, B) \in \Omega,|A|>|B| \Leftrightarrow(x, A) \succ(x, B)$.

Now, for all $x, y \in X$, it follows from (INS) that $(x,\{x\}) \sim\{y,\{y\})$. For all $z \in X \backslash\{x, y\}$, by (IND), $(x,\{x, z\}) \sim(y,\{y, z\})$. It follows from (T3.2) and the transitivity of $\succeq$ that $(x,\{x, y\}) \sim(y,\{x, y\})$. By the repeated use of (T3.2), (IND) and the transitivity of $\succeq$ and noting that opportunity sets are finite, we can show that

(T3.3) For all $(x, A),(y, B) \in \Omega,(x, A) \succeq(y, B) \Leftrightarrow|A| \geq|B|$.

Theorem 3 is thus proved.

Theorem 4. $\succeq$ satisfies (IND), (SI) and (SPO) if and only if it is strongly nonconsequential.

Proof. It can be checked easily that, if $\succeq$ is strongly non-consequential, then it 
satisfies (IND), (SI) and (SPO). Therefore, we have only to prove that, if $\succeq$ satisfies (IND), (SI) and (SPO), then, for all $(x, A),(y, B) \in \Omega,|A|>|B| \Rightarrow(x, A) \succ(y, B)$ and $|A|=|B| \Rightarrow[(x,\{x\}) \succeq(y,\{y\}) \Leftrightarrow(x, A) \succeq(y, B)]$.

Let $\succeq$ satisfy (IND), (SI) and (SPO). By Proposition 6 and following a similar proof method, we can establish that

(T4.1) For all $(x, A),(x, B) \in \Omega,|A|>|B| \Leftrightarrow(x, A) \succ(x, B)$.

For all distinct $x, y \in X$, it follows from (SPO) that $(x,\{x, y\}) \succ(y,\{y\})$. Then, from (T4.1) and by the transitivity of $\succeq,(x,\{x, z\}) \succ(y,\{y\})$ holds for all $z \in$ $X \backslash\{x, y\}$. By virtue of (IND), from $(x,\{x, y\}) \succ(y,\{y\}),(x,\{x, y, z\}) \succ(y,\{y, z\})$ holds for all $z \in X \backslash\{x, y\}$. From (T4.1) and by the transitivity of $\succeq$, we obtain the following:

(T4.2) For all $(x, A),(y, B) \in \Omega$, if $|A|=|B|+1$ and $|B| \leq 2$, then $(x, A) \succ(y, B)$.

From (T4.2), by the repeated use of (IND), (T4.1) and the transitivity of $\succeq$, coupled with the finiteness of opportunity sets, the following can be established:

(T4.3) For all $(x, A),(y, B) \in \Omega$, if $|A|=|B|+1$, then $(x, A) \succ(y, B)$.

From (T4.3), by the transitivity of $\succeq$ and (T4.1), we have

(T4.4) For all $(x, A),(y, B) \in \Omega$, if $|A|>|B|$, then $(x, A) \succ(y, B)$.

Consider now $(x,\{x\})$ and $(y,\{y\})$. If $(x,\{x\}) \sim\{y,\{y\})$, following a similar argument as in the proof of Theorem 4 , we obtain

(T4.5) For all $(x, A),(y, B) \in \Omega$, if $(x,\{x\}) \sim(y,\{y\})$ and $|A|=|B|$, then $(x, A) \sim$ $(y, B)$.

If, on the other hand, $(x,\{x\}) \succ\{y,\{y\})$, we can then follow a similar argument as in the proof of Theorem 2 to obtain

(T4.6) For all $(x, A),(y, B) \in \Omega$, if $(x,\{x\}) \succ(y,\{y\})$ and $|A|=|B|$, then $(x, A) \succ$ $(y, B)$.

(T4.6), together with (T4.4) and (T4.5), completes the proof. 
The following two propositions show, respectively, the independence of the axioms used in Theorems 3 and 4.

Proposition 7. Let $\succeq$ be an ordering. Then the axioms (IND), (SI), (LSM) and (INS) are independent.

Proof. We construct four examples to prove the proposition. In these examples, the respective axiom that is violated is indicated. Let $X=\{x, y, z\}$.

$(\mathrm{P} 7.1) \neg(\mathrm{IND}): \succeq=\succeq_{1}$.

$(\mathrm{P} 7.2) \neg(\mathrm{SI}): \succeq=\succeq_{4}$.

(P7.3) $\neg(\mathrm{LSM}): \succeq=\succeq_{5}$.

(P7.4) $\neg$ (INS): Define $\succeq_{7}$ by $(x, X) \succ_{7}(x,\{x, z\}) \sim_{7}(x,\{x, y\}) \succ_{7}\left\{(x,\{x\}) \succ_{7}\right.$ $(y, X) \succ_{7}(y,\{x, y\}) \sim_{7}(y,\{y, z\}) \succ_{7}(y,\{y\}) \succ_{7}(z, X) \succ_{7}(z,\{x, z\}) \sim_{7}(z,\{y, z\})$ $\succ_{7}(z,\{z\})$. Clearly, $\succeq_{7}$ satisfies (SI), (IND) and (LSM), but it violates (INS) since $(x,\{x\}) \succ_{7}(y,\{y\}) \succ_{7}(z,\{z\})$.

Proposition 8. Let $\succeq$ be an ordering. Then the axioms (IND), (SI) and (SPO) are independent.

Proof. We construct three examples to prove the proposition. In these examples, the respective axiom that is violated is indicated. Let $X=\{x, y, z\}$.

$(\mathrm{P} 8.1) \neg(\mathrm{IND}):$ Define $\succeq_{8}$ by $(x, X) \sim_{8}(y, X) \sim_{8}(z, X) \succ_{8}(x,\{x, y\}) \sim_{8}$ $(x,\{x, z\}) \succ_{8}(y,\{x, y\}) \sim_{8}(y,\{y, z\}) \succ_{8}(z,\{x, z\}) \sim_{8}(z,\{y, z\}) \succ_{8}(x,\{x\}) \sim_{8}$ $(y,\{y\}) \sim_{8}(z,\{z\}) . \succeq_{8}$ satisfies (SI) and (SPO), but it violates (IND) since $(x,\{x\}) \sim_{8}$ $(y,\{y\})$ and $(x,\{x, z\}) \succ_{8}(y,\{y, z\})$.

$(\mathrm{P} 8.2) \neg(\mathrm{SI}):$ Define $\succeq_{9}$ by $(x, X) \succ_{9}(y, X) \sim_{9}(z, X) \succ_{9}(x,\{x, y\}) \succ_{9}(x,\{x, z\})$ $\succ_{9}(y,\{x, y\}) \sim_{9}(y,\{y, z\}) \sim_{9}(z,\{z, x\}) \sim_{9}(z,\{z, y\}) \succ_{9}(x,\{x\}) \succ_{9}(y,\{y\}) \sim_{9}$ $(z,\{z\}) . \succeq_{9}$ satisfies (IND) and (SPO), but it violates (SI) since $(x,\{x, y\})$ $\succ_{9}(x,\{x, z\})$.

$(\mathrm{P} 8.3) \neg(\mathrm{SPO}):$ Define $\succeq_{10}$ by $(x, X) \succ_{10}(x,\{x, y\}) \sim_{10}(x,\{x, z\}) \succ_{10}(x,\{x\}) \succ_{10}$ $(y, X) \succ_{10}(y,\{x, y\}) \sim_{10}(y,\{y, z\}) \succ_{10}(y,\{y\}) \succ_{10}(z, X) \succ_{10}(z,\{z, y\}) \sim_{10}$ $(z,\{x, z\}) \succ_{10}(z,\{z\})$. Then, $\succeq_{10}$ satisfies (IND) and (SI), but it violates (SPO) since $(x,\{x\}) \succ_{10}(y,\{x, y\})$. 


\section{Active Interactions between Outcomes and Op- portunities: The Case of Finite $X$}

So far, we have focused exclusively on the simple special cases where no trade-off exists between consequential considerations, which reflects the decision-making agent's concern about culmination outcomes, and non-consequential considerations, which reflects his concern about richness of opportunities from which culmination outcomes are chosen. For these simple special cases, we have characterized the concepts of consequentialism and non-consequentialism. In this section, we generalize our previous framework by accommodating the situations where consequential considerations and non-consequential considerations actively interact.

Let $\mathcal{Z}$ and $\mathcal{R}$ denote the set of all positive integers and the set of all real numbers, respectively. We first state and prove the following result.

Theorem 5. (Suzumura and $\mathrm{Xu}$ (2003), Theorem 3.3.) Suppose $X$ is finite. $\succeq$ satisfies (IND), (SI) and (SM) if and only if there exist a function $u: X \rightarrow \mathcal{R}$ and a function $f: \mathcal{R} \times \mathcal{Z} \rightarrow \mathcal{R}$ such that

(T5.1) For all $x, y \in X, u(x) \geq u(y) \Leftrightarrow(x,\{x\}) \succeq(y,\{y\})$;

(T5.2) For all $(x, A),(y, B) \in \Omega,(x, A) \succeq(y, B) \Leftrightarrow f(u(x),|A|) \geq f(u(y),|B|)$;

(T5.3) $f$ is non-decreasing in each of its arguments and has the following property: For all integers $i, j, k \geq 1$ and all $x, y \in X$, if $i+k, j+k \leq \# X$, then

(T5.3.1) $f(u(x), i) \geq f(u(y), j) \Leftrightarrow f(u(x), i+k) \geq f(u(y), j+k)$.

The function $u$ in Theorem 5 can be regarded as the usual utility function defined on the set of (conventional) social states, whereas the cardinality of opportunity sets may be regarded as an index of the richness of opportunities offered by opportunity sets. The function $f$ thus weighs the utility of consequential states against the value of richness of opportunities. The active interactions between the utility of consequential states and the value of richness of opportunities are therefore captured by Theorem 5 . It is clear that, the concepts of consequentialsm and non-consequentialism can be obtained as special cases of Theorem 5 by defining the appropriate $f$ functions.

Proof of Theorem 5. We first check the necessity part of the theorem. Suppose 
$u: X \rightarrow \mathcal{R}$ and $f: \mathcal{R} \times \mathcal{Z} \rightarrow \mathcal{R}$ are such that (T5.1), (T5.2) and (T5.3) of Theorem 5 are satisfied.

(SI): Let $x \in X$ and $y, z \in X \backslash\{x\}$. Note that $|\{x, y\}|=|\{x, z\}|$. Therefore, $f(u(x),|\{x, y\}|)=f(u(x),|\{x, z\}|)$, which implies that $(x,\{x, y\}) \sim(x,\{x, z\})$ is true.

(SM): Let $(x, A),(x, B) \in \Omega$ be such that $B \subset A$. Then, $f(u(x),|A|) \geq f(u(x),|B|)$ holds, since $f$ is non-decreasing in each of its arguments and $|A| \geq|B|$. Therefore, $(x, A) \succeq(x, B)$.

(IND): Let $(x, A),(y, B) \in \Omega$, and $z \in X \backslash A \cup B$. From (T5.3.1), we have

$f(u(x),|A|) \geq f(u(y),|B|) \Leftrightarrow f(u(x),|A|+1) \geq f(u(y),|B|+1)$

$\Leftrightarrow f(u(x),|(A \cup\{z\})|) \geq f(u(y),|(B \cup\{z\})|)$.

Therefore, $(x, A) \succeq(y, B) \Leftrightarrow(x, A \cup\{z\}) \succeq(y, B \cup\{z\})$.

Next, we show that, if $\succeq$ satisfies (IND), (SI) and (SM), then there exist a function $f: \mathcal{R} \times \mathcal{Z} \rightarrow \mathcal{R}$ and a function $u: X \rightarrow \mathcal{R}$ such that (T5.1), (T5.2) and (T5.3) of Theorem 5 hold. Let $\succeq$ satisfy (IND), (SI) and (SM). Note that, $X$ is finite and so is $\Omega$. The ordering of $\succeq$ implies that there exist $u: X \rightarrow \mathcal{R}$ and $F: \Omega \rightarrow \mathcal{R}$ such that

(T5.4) For all $x, y \in X,(x,\{x\}) \succeq(y,\{y\}) \Leftrightarrow u(x) \geq u(y)$;

(T5.5) For all $(x, A),(y, B) \in \Omega,(x, A) \succeq(y, B) \Leftrightarrow F(x, A) \geq F(y, B)$.

(T5.1) of Theorem 5 then follows immediately. To show that (T5.2) holds, let $(x, A),(y, B) \in \Omega$ be such that $u(x)=u(y)$ and $|A|=|B|$. From $u(x)=u(y)$, we must have $(x,\{x\}) \sim(y,\{y\})$. Then, by making repeated use of Proposition 3 if necessary and noting that $|A|=|B|,(x, A) \sim(y, B)$ can be obtained easily. Define $\Sigma \subset \mathcal{R} \times \mathcal{Z}$ as follows: $\Sigma:=\{(t, i) \in \mathcal{R} \times \mathcal{Z} \mid \exists(x, A) \in \Omega: t=u(x)$ and $i=|A|\}$. Next, define a binary relation $\geq^{*}$ on $\Sigma$ as follows: For all $(x, A),(y, B) \in \Omega$, $(x, A) \succeq(y, B) \Leftrightarrow(u(x),|A|) \geq^{*}(u(y),|B|)$. From the above discussion and noting that $\succeq$ satisfies (SM) and (IND), the binary relation $\geq^{*}$ defined on $\Sigma$ is an ordering, and it has the following properties:

$\left(\mathrm{SM}^{\prime}\right)$ : For all $(t, i),(t, j) \in \Sigma$, if $j \geq i$ then $(t, j) \geq^{*}(t, i)$;

$\left(\operatorname{IND}^{\prime}\right)$ : For all $(s, i),(t, j) \in \Sigma$, and all integer $k$, if $i+k \leq|X|$ and $j+k \leq|X|$, then $(s, i) \geq^{*}(t, j) \Leftrightarrow(s, i+k) \geq^{*}(t, j+k)$.

Since $\Sigma$ is finite and $\geq^{*}$ is an ordering on $\Sigma$, there exists a function $f: \mathcal{R} \times \mathcal{Z} \rightarrow \mathcal{R}$ such that, for all $(s, i),(t, j) \in \Sigma,(s, i) \geq^{*}(t, j)$ iff $f(s, i) \geq f(t, j)$. From the 
definition of $\geq^{*}$ and $\Sigma$, we must have the following: For all $(x, A),(y, B) \in \Omega,(x, A) \succeq$ $(y, B) \Leftrightarrow(u(x),|A|) \geq^{*}(u(y),|B|) \Leftrightarrow f(u(x),|A|) \geq f(u(y),|B|)$. To prove that $f$ is non-decreasing in each of its arguments, we first consider the case in which $u(x) \geq u(y)$ and $|A|=|B|$. Given $u(x) \geq u(y)$, it follows from the definition of $u$ that $(x,\{x\}) \succeq(y,\{y\})$. Noting that $|A|=|B|$, by the repeated use of Proposition 3 , if necessary, we must have $(x, A) \succeq(y, B)$. Thus, $f$ is non-decreasing in its first argument. To show that $f$ is non-decreasing in its second argument as well, we consider the case in which $u(x)=u(y)$ and $|A| \geq|B|$. From $u(x)=u(y)$, we must have $(x,\{x\}) \sim(y,\{y\})$. Then, from the earlier argument, $\left(x, A^{\prime}\right) \sim(y, B)$ for some $A^{\prime} \subset A$ such that $\left|A^{\prime}\right|=|B|$. Now, by (SM), $(x, A) \succeq\left(x, A^{\prime}\right)$. Then, $(x, A) \succeq(y, B)$ follows from the transitivity of $\succeq$. Therefore, $f$ is non-decreasing in each of its arguments. Finally, (T5.3.1) follows clearly from $\left(\mathrm{IND}^{\prime}\right)$.

A limitation of Theorem 5 is that it assumes $X$ to be finite. In many contexts in economics, the universal set of social state is typically infinite. The following two results deal with this case: Theorem 6 presents a full characterization of all the orderings satisfying (IND), (SI) and (SM), while Theorem 7 gives a representation of any ordering characterized in Theorem 6 .

Theorem 6. (Suzumura and $\mathrm{Xu}$ (2003) Theorem 4.1) $\succeq$ satisfies (IND), (SI) and (SM) if and only if there exists an ordering $\succeq \#$ on $X \times \mathcal{Z}$ such that

(T6.1) For all $(x, A),(y, B) \in \Omega,(x, A) \succeq(y, B) \Leftrightarrow(x,|A|) \succeq \#(y,|B|)$;

(T6.2) For all integers $i, j, k \geq 1$ and all $x, y \in X,(x, i) \succeq^{\#}(y, j) \Leftrightarrow(x, i+k) \succeq^{\#}$ $(y, j+k)$, and $(x, i+k) \succeq \#(x, i)$.

Proof. Let $\succeq$ satisfy (IND), (SI) and (SM). By Proposition 1, we have,

(T6.3) For all $(a, A),(a, B) \in \Omega$, if $|A|=|B|$, then $(a, A) \sim(a, B)$.

By Proposition 3, the following can be shown to be true:

(T6.4) For all $x, y \in X$ and all $(x, A),(y, A) \in \Omega,(x,\{x\}) \succeq(y,\{y\}) \Leftrightarrow(x, A) \succeq$ $(y, A)$.

We now show that, for all $(x, A),(y, B) \in \Omega$, if $(x,\{x\}) \sim(y,\{y\})$ and $|A|=|B|$, then $(x, A) \sim(y, B)$. Let $C \in K$ be such that $|C|=|A|=|B|$ and $\{x, y\} \subset C$. From 
(T6.4), we have $(x, C) \sim(y, C)$. Note that $(x, C) \sim(x, A)$ and $(y, C) \sim(y, B)$ follow from (T6.3). By transitivity of $\sim$, we have $(x, A) \sim(y, B)$. Define a binary relation $\succeq^{\#}$ on $X \times \mathcal{Z}$ as follows: For all $x, y \in X$ and all positive integers $i, j,(x, i) \succeq$ \# $(y, j) \Leftrightarrow[(x, A) \succeq(y, B)$ for some $A, B \in K$ such that $x \in A, y \in B, i=|A|, j=|B|]$. From the above discussion, $\succeq^{\#}$ is well-defined and is an ordering. A similar method of proving Theorem 5 (T5.3) can be invoked to prove that (T6.2) holds.

To present our next theorem, we need the following continuity property, which was introduced in Suzumura and Xu (2003). Suppose that $X=\mathcal{R}_{+}^{n}$ for some natural number $n$.

Continuity (CON): For all $(x, A) \in \Omega$, all $y, y^{i} \in X(i=1,2, \cdots)$ and all $B \in$ $K \cup\{\emptyset\}$, if $B \cap\left\{y^{i}\right\}=B \cap\{y\}=\emptyset$ for all $i=1,2, \cdots$, and $\lim _{i \rightarrow \infty} y^{i}=y$, then $\left[\left(y^{i}, B \cup\left\{y^{i}\right\}\right) \succeq(x, A)\right.$ for $\left.i=1,2, \cdots\right] \Rightarrow(y, B \cup\{y\}) \succeq(x, A)$, and $\left[(x, A) \succeq\left(y^{i}, B \cup\left\{y^{i}\right\}\right)\right.$ for $\left.i=1,2, \cdots\right] \Rightarrow(x, A) \succeq(y, B \cup\{y\})$.

Theorem 7. (Suzumura and Xu (2003), Theorem 4.5) Suppose that $X=\mathcal{R}_{+}^{n}$ and that $\succeq$ satisfies (IND), (SI), (SM) and (CON). Then, there exists a function $v: X \times \mathcal{Z} \rightarrow \mathcal{R}$, which is continuous in its first argument, such that

(T7.1) For all $(x, A),(y, B) \in \Omega,(x, A) \succeq(y, B) \Leftrightarrow v(x,|A|) \geq v(y,|B|)$,

(T7.2) For all $i, j, k \in \mathcal{Z}$ and all $x, y \in X, v(x, i) \geq v(y, j) \Leftrightarrow v(x, i+k) \geq v(y, j+k)$ and $v(x, i+k) \geq v(x, i)$.

Proof. From Theorem 6, we know that there exists an ordering $\succeq^{\#}$ on $X \times \mathcal{Z}$ such that

(T7.3) For all $(x, A),(y, B) \in \Omega,(x, A) \succeq(y, B) \Leftrightarrow(x,|A|) \succeq^{\#}(y,|B|)$;

(T7.4) For all integers $i, j, k \geq 1$ and all $x, y \in X,(x, i) \succeq^{\#}(y, j) \Leftrightarrow(x, i+k) \succeq^{\#}$ $(y, j+k) ;$ and $(x, i+k) \succeq^{\#}(x, i)$.

For all $(x, i) \in X \times \mathcal{Z}$ and all $j \in \mathcal{Z}$, consider the sets $U(x ; i, j)$ and $L(x ; i, j)$ defined as follows:

$$
U(x ; i, j)=\left\{y \in X \mid(y, j) \succeq^{\#}(x, i)\right\}, L(x ; i, j)=\left\{y \in X \mid(x, i) \succeq^{\#}(y, j)\right\} .
$$


From (T7.3), by (CON), it is clear that both $U(x ; i, j)$ and $L(x ; i, j)$ are closed. Note that $X=\mathcal{R}_{+}^{n}$. By Theorem 3 of Blackorby, Bossert, and Donaldson (2001), there is a function $v: X \times \mathcal{Z} \rightarrow \mathcal{R}$, which is continuous in its first argument, that represents $\succeq \#$. Therefore, the part (T7.1) of Theorem 7 obtains. The part (T7.2) of Theorem 7 follows from (T7.1), (T7.3) and (T7.4).

\section{Applications}

\subsection{Arrovian Social Choice}

In this subsection, we discuss how our notions of consequentialism and nonconsequentialism can affect the fate of Arrow's impossibility theorem in social choice theory. For this purpose, let $X$ consist of at least three but finite social alternatives. Each alternative in $X$ is assumed to be a public alternative such as a list of public goods to be provided in the society, or the description of a candidate in the public election. The set of all individuals in the society is denoted by $N=\{1,2, \ldots, n\}$, where $+\infty>n \geq 2$. Each individual $i \in N$ is assumed to have an extended preference ordering $R_{i}$ over $\Omega$, which is reflexive, complete and transitive. For any $(x, A),(y, B) \in \Omega,(x, A) R_{i}(y, B)$ is interpreted as follows: $i$ feels at least as good when choosing $x$ from $A$ as when choosing $y$ from $B$. The asymmetric part and the symmetric part of $R_{i}$ are denoted by $P\left(R_{i}\right)$ and $I\left(R_{i}\right)$, respectively, which denote the strict preference relation and the indifference relation of $i \in N$.

The set of all logically possible orderings over $\Omega$ is denoted by $\mathcal{R}$. Then, a profile $\mathbf{R}=\left(R_{1}, R_{2}, \cdots, R_{n}\right)$ of extended individual preference orderings, one extended ordering for each individual, is an element of $\mathcal{R}^{n}$. An extended social welfare function (ESWF) is a function $f$ which maps each and every profile in some subset $D_{f}$ of $\mathcal{R}^{n}$ into $\mathcal{R}$. When $R=f(\mathbf{R})$ holds for some $\mathbf{R} \in D_{f}, I(R)$ and $P(R)$ stand, respectively, for the social indifference relation and the social strict preference relation corresponding to $R$. Given an $\operatorname{ESWF} f$, the problem of social choice we consider in this subsection can be described as follows. Suppose that a profile $\mathbf{R} \in D_{f}$ and a set $S \subseteq X$ of feasible social alternatives are given. Then the best social choice from $S$ can be identified to be an $x^{*} \in S$ such that $\left(x^{*}, S\right) R(x, S)$ holds for all $x \in S$, where $R=f(\mathbf{R})$.

We assume that each and every profile $\mathbf{R}=\left(R_{1}, R_{2}, \cdots, R_{n}\right) \in D_{f}$ is such that 
$R_{i}$ satisfies the properties (IND), (SI) and (SM) for all $i \in N$.

In addition to the domain restriction on $D_{f}$ introduced above, we first introduce two conditions corresponding to Arrow's (1963) Pareto principle and Non-dictatorship to be imposed on $f$. They are well known, and require no further explanation.

Strong Pareto Principle (SP): For all $(x, A),(y, B) \in \Omega$, and for all $\mathbf{R}=$ $\left(R_{1}, R_{2}, \cdots, R_{n}\right) \in D_{f}$, if $(x, A) P\left(R_{i}\right)(y, B)$ holds for all $i \in N$, then we have $(x, A) P(R)(y, B)$, and if $(x, A) I\left(R_{i}\right)(y, B)$ holds for all $i \in N$, then we have $(x, A) I(R)(y, B)$, where $R=f(\mathbf{R})$.

Non-Dictatorship (ND): There exists no $i \in N$ such that $\left[(x, A) P\left(R_{i}\right)(y, B) \Rightarrow\right.$ $(x, A) P(R)(y, B)$ for all $(x, A),(y, B) \in \Omega]$ holds for all $\mathbf{R}=\left(R_{1}, R_{2}, \cdots, R_{n}\right) \in$ $D_{f}$, where $R=f(\mathbf{R})$.

There are various ways of formulating Arrow's IIA in our context. Consider the following:

Independence of Irrelevant Alternatives (i) (IIA(i)): For all $\mathbf{R}^{\mathbf{1}}=\left(R_{1}^{1}, R_{2}^{1}\right.$, $\left.\cdots, R_{n}^{1}\right), \mathbf{R}^{2}=\left(R_{1}^{2}, R_{2}^{2}, \cdots, R_{n}^{2}\right) \in D_{f}$, and for all $(x, A),(y, B) \in \Omega$, if $\left[(x, A) R_{i}^{1}(y, B)\right.$ $\Leftrightarrow(x, A) R_{i}^{2}(y, B)$ and $\left.(x,\{x\}) R_{i}^{1}(y,\{y\}) \Leftrightarrow(x,\{x\}) R_{i}^{2}(y,\{y\})\right]$ holds for all $i \in N$, then $\left[(x, A) R^{1}(y, B) \Leftrightarrow(x, A) R^{2}(y, B)\right]$ holds, where $R^{1}=f\left(\mathbf{R}^{\mathbf{1}}\right)$ and $R^{2}=f\left(\mathbf{R}^{2}\right)$.

Independence of Irrelevant Alternatives (ii) (IIA(ii)): For all $\mathbf{R}^{\mathbf{1}}=\left(R_{1}^{1}, R_{2}^{1}\right.$, $\left.\cdots, R_{n}^{1}\right), \mathbf{R}^{2}=\left(R_{1}^{2}, R_{2}^{2}, \cdots, R_{n}^{2}\right) \in D_{f}$, and for all $(x, A),(y, B) \in \Omega$ with $|A|=|B|$, if $\left[(x, A) R_{i}^{1}(y, B) \Leftrightarrow(x, A) R_{i}^{2}(y, B)\right]$ holds for all $i \in N$, then $\left[(x, A) R^{1}(y, B) \Leftrightarrow(x, A) R^{2}(y, B)\right]$ holds, where $R^{1}=f\left(\mathbf{R}^{\mathbf{1}}\right)$ and $R^{2}=f\left(\mathbf{R}^{\mathbf{2}}\right)$.

Full Independence of Irrelevant Alternatives (FIIA): For all $\mathbf{R}^{\mathbf{1}}=$ $\left(R_{1}^{1}, R_{2}^{1}, \cdots, R_{n}^{1}\right), \mathbf{R}^{2}=\left(R_{1}^{2}, R_{2}^{2}, \cdots, R_{n}^{2}\right) \in D_{f}$, and for all $(x, A),(y, B) \in \Omega$, if $\left[(x, A) R_{i}^{1}(y, B) \Leftrightarrow(x, A) R_{i}^{2}(y, B)\right]$ holds for all $i \in N$, then $\left[(x, A) R^{1}(y, B) \Leftrightarrow\right.$ $\left.(x, A) R^{2}(y, B)\right]$ holds, where $R^{1}=f\left(\mathbf{R}^{1}\right)$ and $R^{2}=f\left(\mathbf{R}^{2}\right)$.

(IIA(i)) says that the extended social preference between any two extended alternatives $(x, A)$ and $(y, B)$ depends on each individual's extended preference between them as well as each individual's extended preference between $(x,\{x\})$ and 
$(y,\{y\})$ : for any two profiles $\mathbf{R}^{1}$ and $\mathbf{R}^{2}$, if, $\left[(x, A) R_{i}^{1}(y, B)\right.$ holds if and only if $(x, A) R_{i}^{2}(y, B)$ holds, and $(x,\{x\}) R_{i}^{1}(y,\{y\})$ holds if and only if $(x,\{x\}) R_{i}^{2}(y,\{y\})$ holds], for all $i \in N$, then $(x, A) R^{1}(y, B)$ holds if and only if $(x, A) R^{2}(y, B)$ holds, where $R^{1}=f\left(\mathbf{R}^{1}\right)$ and $R^{2}=f\left(\mathbf{R}^{2}\right)$. (IIA(ii)), on the other hand, says that the extended social preference between any two extended alternatives $(x, A)$ and $(y, B)$ with $|A|=|B|$ depends on each individual's extended preference between them. Finally, (FIIA) says that, the extended social preference between any two extended alternatives $(x, A)$ and $(y, B)$ depends on each individual's extended preference between them. It is clear that (IIA(i)) is logically independent of (IIA(ii)), and both (IIA(i)) and (IIA(ii)) are logically weaker than (FIIA).

Let us observe that each and every individual in the original Arrow framework can be regarded as an extreme consequentialist. Thus, Arrow's impossibility theorem can be viewed as an impossibility result in the framework of extreme consequentialism. What will happen to the impossibility theorem in a framework which is broader than the extreme consequentialism? For the purpose of answering this question, let us now introduce three domain restrictions on $f$ by specifying some appropriate subsets of $D_{f}$. In the first place, let $D_{f}(E)$ be the set of all profiles in $D_{f}$ such that all individuals are extreme consequentialists. Secondly, let $D_{f}(E \cup S)$ be the set of all profiles in $D_{f}$ such that at least one individual is an extreme consequentialist uniformly for all profiles in $D_{f}(E \cup S)$ and at least one individual is a strong consequentialist uniformly for all profiles in $D_{f}(E \cup S)$. Finally, let $D_{f}(N)$ be the set of all profiles in $D_{f}$ such that at least one individual is a strong non-consequentialist uniformly for all profiles in $D_{f}(N)$.

Our first result in this subsection is nothing but a restatement of Arrow's original impossibility theorem in the framework of extreme consequentialism.

Theorem 8. Suppose that all individuals are extreme consequentialists. Then, there exists no extended social welfare function $f$ with the domain $D_{f}(E)$ which satisfies (SP), (ND), and either (IIA(i)) or (IIA(ii)).

Proof. Suppose that there exists an ESWF $f$ on $D_{f}(E)$ which satisfies (SP) and (IIA(i)). Since all individuals are extreme consequentialists,

$(\mathrm{T} 8.1) \forall i \in N:(x, A) R_{i}(y, B) \Leftrightarrow(x, X) R_{i}(y, X)$

holds for all $(x, A),(y, B) \in \Omega$ and for all $\mathbf{R}=\left(R_{1}, R_{2}, \cdots, R_{n}\right) \in D_{f}(E)$. Note 
that the conditions (IND), (SI) and (SM) impose no restriction whatsoever on the profile $\mathbf{R}=\left(R_{1}, R_{2}, \cdots, R_{n}\right)$ even when, for each and every $i \in N, R_{i}$ is restricted on $\Omega_{X}:=\{(x, X) \in X \times K \mid x \in X\}$. Note also that (SP) and (IIA(i)) imposed on $f$ imply that the same conditions must be satisfied on the restricted space $\Omega_{X}$. By virtue of the Arrow impossibility theorem, therefore, there exists a dictator, say $d \in N$, for $f$ on the restricted space $\Omega_{X}$. That is, for all $\mathbf{R}=\left(R_{1}, R_{2}, \cdots, R_{n}\right) \in D_{f}(E)$ and all $(x, X),(y, X) \in \Omega_{X},(x, X) P\left(R_{d}\right)(y, X) \Rightarrow(x, X) P(R)(y, X)$, where $R=f(\mathbf{R})$. We now show that for all $(x, A),(y, B) \in \Omega,(x, A) P\left(R_{d}\right)(y, B) \Rightarrow(x, A) P(R)(y, B)$, viz., $d$ is a dictator for $f$ on the full space $\Omega$. Note that, since $d$ is an extreme consequentialist, we must have that $(x, A) P\left(R_{d}\right)(y, B)$ iff $(x, X) P\left(R_{d}\right)(y, X)$. Since all individuals are extreme consequentialists, it must be true that $(x, A) I\left(R_{i}\right)(x, X)$ and $(y, B) I\left(R_{i}\right)(y, X)$ for all $i \in N$. Therefore, by $(\mathrm{SP}),(x, A) I(R)(x, X)$ and $(y, B) I(R)(y, X)$. By virtue of the transitivity of $R$, it then follows that $(x, X) P(R)(y$, $X) \Rightarrow(x, A) P(R)(y, B)$. That is, we have shown that $(x, A) P\left(R_{d}\right)(y, B) \Rightarrow(x, A) P(R)$ $(y, B)$. In other words, $d$ is a dictator for $f$ on the full space $\Omega$. Therefore, there exists no ESWF that satisfies (SP), (IIA(i)) and (ND).

A similar argument as above can be used to show that there exists no ESWF that satisfies (SP), (IIA(ii)) and (ND).

However, once we go beyond the framework of extreme consequentialism, as shown by the following results, there is a scope for resolving the impossibility result.

Theorem 9. Suppose that there exist at least one uniform extreme-consequentialist over $D_{f}(E \cup S)$ and at least one uniform strong-consequentialist over $D_{f}(E \cup S)$ in the society. Then, there exists an extended social welfare function $f$ with the domain $D_{f}(E \cup S)$ satisfying (SP), (IIA(i)), (IIA(ii)) and (ND).

Proof. Let $e \in N$ be a uniform extreme-consequentialist and $s \in N$ be a uniform strong-consequentialist. By definition,

(T9.1) $\forall(x, A),(x, B) \in \Omega:(x, A) I\left(R_{e}\right)(x, B)$,

$(\mathrm{T} 9.2) \forall(x, A),(y, B) \in \Omega:(x,\{x\}) I\left(R_{s}\right)(y,\{y\}) \Rightarrow\left[(x, A) R_{s}(y, B) \Leftrightarrow|A| \geq|B|\right]$,

and

$(\mathrm{T} 9.3) \forall(x, A),(y, B) \in \Omega:(x,\{x\}) P\left(R_{s}\right)(y,\{y\}) \Rightarrow(x, A) P\left(R_{s}\right)(y, B)$ 
hold. Now consider the following ESWF:

$$
\begin{aligned}
& \forall(x, A),(y, B) \in \Omega: \\
& (x,\{x\}) P\left(R_{s}\right)(y,\{y\}) \Rightarrow\left[(x, A) R(y, B) \Leftrightarrow(x, A) R_{s}(y, B)\right] ; \\
& (x,\{x\}) I\left(R_{s}\right)(y,\{y\}) \Rightarrow\left[(x, A) R(y, B) \Leftrightarrow(x, A) R_{e}(y, B)\right], \\
& \text { where } R=f(\mathbf{R}) .
\end{aligned}
$$

It may easily be verified that the above ESWF satisfies (SP) and (ND). To verify that it satisfies both (IIA(i)) and (IIA(ii)), we consider $(x, A),(y, B) \in \Omega$, and $\mathbf{R}=$ $\left(R_{1}, R_{2}, \cdots, R_{n}\right), \mathbf{R}^{\prime}=\left(R_{1}^{\prime}, R_{2}^{\prime}, \cdots, R_{n}^{\prime}\right) \in D_{f}(E \cup S)$. Let $R=f(\mathbf{R})$ and $R^{\prime}=f\left(\mathbf{R}^{\prime}\right)$.

To begin with, suppose that we have $(x, A) R_{i}(y, B) \Leftrightarrow(x, A) R_{i}^{\prime}(y, B)$ as well as $(x,\{x\}) R_{i}(y,\{y\}) \Leftrightarrow(x,\{x\}) R_{i}^{\prime}(y,\{y\})$ for all $i \in N$. If $(x,\{x\}) P\left(R_{s}\right)(y,\{y\})$, then $(x,\{x\}) P\left(R_{s}^{\prime}\right)(y,\{y\}),(x, A) P\left(R_{s}\right)(y, B)$, as well as $(x, A) P\left(R_{s}^{\prime}\right)(y, B)$. Then, the ESWF gives us $(x, A) P(R)(y, B)$ and $(x, A) P\left(R^{\prime}\right)(y, B)$. Secondly, if $(y,\{y\}) P\left(R_{s}\right)(x$, $\{x\})$, then $(y,\{y\}) P\left(R_{s}^{\prime}\right)(x,\{x\}),(y, B) P\left(R_{s}\right)(x, A)$, and $(y, B) P\left(R_{s}^{\prime}\right)(x, A)$. Then, the ESWF gives us $(y, B) P(R)(x, A)$ and $(y, B) P\left(R^{\prime}\right)(x, A)$. Thirdly, if $(x,\{x\}) I\left(R_{s}\right)$ $(y,\{y\})$, then $(x,\{x\}) I\left(R_{s}^{\prime}\right)(y,\{y\})$. The ESWF implies that $(x, A) R(y, B) \Leftrightarrow(x, A) R_{e}$ $(y, B)$ and $(x, A) R^{\prime}(y, B) \Leftrightarrow(x, A) R_{e}^{\prime}(y, B)$. Note that individual $e$ is an extreme consequentialist. It is therefore clear that, in this case, if $(x, A) R_{e}(y, B) \Leftrightarrow(x, A) R_{e}^{\prime}(y, B)$, then $(x, A) R(y, B) \Leftrightarrow(x, A) R^{\prime}(y, B)$. Therefore, (IIA(i)) is satisfied.

Next, suppose that $|A|=|B|$ and that $\left[(x, A) R_{i}(y, B) \Leftrightarrow(x, A) R_{i}^{\prime}(y, B)\right]$ for all $i \in N$. To show that $(x, A) R(y, B) \Leftrightarrow(x, A) R^{\prime}(y, B)$ in this case, we observe that, when $|A|=|B|,(x, A) R_{s}(y, B) \Leftrightarrow(x,\{x\}) R_{s}(y,\{y\})$ and $(x, A) R_{s}^{\prime}(y, B) \Leftrightarrow$ $(x,\{x\}) R_{s}^{\prime}(y,\{y\})$. Then the proof that the above ESWF satisfies (IIA(ii)) is similar to the proof showing that the ESWF satisfies (IIA(i)). We have only to note that the individual $e$ is an extreme consequentialist.

The binary relation $R$ generated by this ESWF is clearly reflexive and complete. We now show that $R$ is transitive. Let $(x, A),(y, B)$ and $(z, C) \in \Omega$ be such that $(x, A) R(y, B)$ and $(y, B) R(z, C)$. Note that, since $(x, A) R(y, B)$, by the ESWF constructed above, we cannot have $(y,\{y\}) P\left(R_{s}\right)(x,\{x\})$. Then, by the completeness of $R_{s}$, there are only two cases to be distinguished and separately considered: (a) $(x,\{x\}) I\left(R_{s}\right)(y,\{y\})$; and (b) $(x,\{x\}) P\left(R_{s}\right)(y,\{y\})$.

Case (a): In this case, we must have $(x, A) R_{e}(y, B)$. If $(y,\{y\}) I\left(R_{s}\right)(z,\{z\})$, then it follows from $(y, B) R(z, C)$ that $(y, B) R_{e}(z, C)$. Then, the transitivity of $R_{e}$ implies $(x, A) R_{e}(z, C)$. By the transitivity of $R_{s},(x,\{x\}) I\left(R_{s}\right)(z,\{z\})$. There- 
fore, $(x, A) R(z, C)$ if and only if $(x, A) R_{e}(z, C)$. Hence, $(x, A) R(z, C)$ follows from $(x, A) R_{e}(z, C)$. If $(y,\{y\}) P\left(R_{s}\right)(z,\{z\})$, then, by the transitivity of $R_{s}$, it follows that $(x,\{x\}) P\left(R_{s}\right)(z,\{z\})$. Therefore, $(x, A) R(z, C)$ if and only if $(x, A) R_{s}(z, C)$. Since $s$ is a strong consequentialist, given that $(x,\{x\}) P\left(R_{s}\right)(z,\{z\})$, we must have $(x, A) P\left(R_{s}\right)(z, C)$. Therefore, $(x, A) P(R)(z, C)$. Hence, $(x, A) R(z, C)$ holds. Note that, given $(y, B) R(z, C)$, we cannot have $(z,\{z\}) P\left(R_{s}\right)(y,\{y\})$. Therefore, the transitivity of $R$ holds in the case (a).

Case (b): In this case, we must have $(x, A) P\left(R_{s}\right)(y, B)$, hence $(x, A) P(R)(y, B)$. Since $(y, B) R(z, C)$, we must then have $(y,\{y\}) R_{s}(z,\{z\})$. By the transitivity of $R_{s}$, it follows that $(x,\{x\}) P\left(R_{s}\right)(z,\{z\})$. Thus, $(x, A) P\left(R_{s}\right)(z, C)$ follows from $s$ being a strong consequentialist. By construction, in this case, $(x, A) R(z, C)$ if and only if $(x, A) R_{s}(z, C)$. Hence, $(x, A) P(R)(z, C)$. Therefore, the transitivity of $R$ holds in the case (b).

Combining the cases (a) and (b), the transitivity of $R$ is proved.

Theorem 10. (Suzumura and Xu (2004), Theorem 4) Suppose that there exists at least one person who is a uniform strong non-consequentialist over $D_{f}(N)$. Then, there exists an extended social welfare function $f$ with the domain $D_{f}(N)$ that satisfies (SP), (FIIA) and (ND).

Proof. Let $n^{*} \in N$ be a uniform strong non-consequentialist over $D_{f}(N)$. Then, for all $\mathbf{R}=\left(R_{1}, R_{2}, \cdots, R_{n}\right) \in D_{f}(N)$ and all $(x, A),(y, B) \in \Omega$, it follows from $|A|>|B|$ that $(x, A) P\left(R_{n^{*}}\right)(y, B)$. Consider now the following ESWF $f$ : For all $(x, A),(y, B) \in \Omega$,

$$
\begin{aligned}
& \text { if }|A|>|B| \text {, then }(x, A) P(R)(y, B) ; \\
& \text { if }|A|=|B|=1 \text {, then }(x,\{x\}) R(y,\{y\}) \text { iff }(x,\{x\}) R_{1}(y,\{y\}) ; \\
& \text { if }|A|=|B|=2 \text {, then }(x, A) R(y, B) \text { iff }(x, A) R_{2}(y, B) ; \\
& \vdots \\
& \text { if } A=B=X \text {, then }(x, A) R(y, A) \text { iff }(x, A) R_{k}(y, B) \text {, where } k=\min \{|N|,|X|\},
\end{aligned}
$$

where $R=f(\mathbf{R})$. It is easy to verify that this $f$ satisfies (SP), (FIIA) and (ND). It is also clear that $R$ generated by this ESWF is reflexive and complete. We now show that $R$ is transitive as well. Let $(x, A),(y, B),(z, C) \in \Omega$ be such that $(x, A) R(y, B)$ and $(y, B) R(z, C)$. Then, clearly, $|A| \geq|B|$ and $|B| \geq|C|$. If $|A|>|B|$ or $|B|>|C|$, 
then $|A|>|C|$. By the constructed ESWF, $(x, A) P(R)(z, C)$ follows easily. Thus, the transitivity of $R$ holds for this case. Now, suppose $|A|=|B|=|C|$. Note that in this case, for all $(a, G),(b, H) \in \Omega$ such that $|G|=|H|=|A|,(a, G) R(b, H)$ iff $(a, G) R_{k}(b, H)$ where $k \in N$ and $k=\min \{|N|,|A|\}$. Therefore, the transitivity of $R$ follows from the transitivity of $R_{k}$. The above two cases exhaust all the possibilities. Therefore, $R$ is transitive.

To conclude this subsection, the following observations may be in order. To begin with, as shown by Iwata (2006), the possibility result obtained in Theorem 9 no longer holds if (IIA(i)) or (IIA(ii)) is replaced by (FIIA), while retaining (SP) and (ND) intact. On the other hand, as reported in Iwata (2006), there exists an ESWF over the domain $D_{f}(E \cup S)$ that satisfies (FIIA), (ND) and (WP): for all $(x, A),(y, B) \in \Omega$, and all $\mathbf{R}=\left(R_{1}, R_{2}, \cdots, R_{n}\right) \in D_{f}(E \cup S)$, if $(x, A) P\left(R_{i}\right)(y, B)$ for all $i \in N$, then $(x, A) P(R)(y, B)$, where $R=F(\mathbf{R})$. The proof of this result is quite involved, and the interested readers are referred to Iwata (2006). Secondly, given that (FIIA) is stronger than (IIA(i)) or (IIA(ii)), the impossibility result of Theorem 8 still holds if (IIA(i)) or (IIA(ii)) is replaced by (FIIA), while retaining (SP) and (ND) intact. Thirdly, since the ESWF constructed in the proof of Theorem 10 satisfies (FIIA), it is clear that there exists an ESWF on $D_{f}(N)$ that satisfies (SP), (ND) and both (IIA(i)) and (IIA(ii)).

\subsection{Ultimatum Games}

In experimental studies of two-player extensive form games with complete information, it is observed that the second mover is not only concerned about his own monetary payoff, but cares also about the feasible set that has emerged from the first mover's choice and from which he must make his choice (see, for example, Cox, Friedman and Gjerstad (2006), and Cox, Friedman and Sadiraj (2005)). For the sake of easy presentation, we shall focus on ultimatum games where two players, the Proposer and the Responder, are to divide a certain amount of money between them, and see what is the framework which naturally suggests itself in this context.

Formally, an ultimatum game consists of two players, the Proposer and the Responder. The sequence of the game is as follows. The Proposer moves first, and he is presented a set $X$ of feasible division rules by the experimenter. A division rule is then chosen by the Proposer from the set $X$, which consists of the division rules in 
the pattern of $(50,50),(80,20),(60,40),(70,30)$, and the like. Proposer chooses a division rule $x \in X$. The intended interpretation is that the Proposer gets $x \%$ and the Responder gets $(1-x) \%$ of the money to be divided. Upon seeing a division rule chosen by the Proposer from the given set $X$, the Responder then chooses an amount $m \geq 0$ of money to be divided between them. As a consequence, the Proposer's monetary payoff is $x m$, and the Responder's monetary payoff is $(1-x) m$. Consider the same payoff 8 for the Proposer and 2 for the Responder derived from two different situations, one involving the Proposer's choice of the $(80,20)$ division rule from the set $\{(80,20)\}$ and the other involving the Proposer's choice of the $(80,20)$ division rule from the set $\{(80,20),(70,30),(60,40),(50,50),(40,60),(30,70),(20,80)\}$, the Responder's choice of money to be divided remaining the same at 10. Though the two situations yield the same payoff vector, the Responder's behavior has been observed to be very different. Though there are several possible explanations for such different behaviors on the Responder's side, we can explain the difference in the Responder's behavior via our notions of consequentialism and non-consequentialism.

Let $x$ be the division rule chosen by the Proposer from the given set $A$ of feasible division rules. The associated payoff vector with the division rule $x \in A$ is denoted by $m(x)=\left(m_{P}(x), m_{R}(x)\right)$, where $m_{P}(x)$ is the Proposer's payoff and $m_{R}(x)$ is the Responder's payoff. In our extended framework, we may describe the situation by the triple, $(m(x), x, A)$ with the interpretation that the payoff vector is $m(x)$ for the chosen division rule $x$ from the feasible set $A$. Let $X$ be the finite set of all possible division rules, and $\Omega$ be the set of all possible triples $(m(x), x, A)$, where $A \subseteq X$ and $x \in A$. Let $\succeq$ be the Responder's preference relation (reflexive and transitive, but not necessarily complete) over $\Omega$ with its symmetric and asymmetric parts denoted, respectively, by $\sim$ and $\succ$. Then, we may define several notions of consequentialism and non-consequentialism. For example, we may say that the Responder is

(i) an extreme consequentialist if, for all $(m(x), x, A),(m(y), y, B) \in \Omega, m(x)=$ $m(y) \Rightarrow(m(x), x, A) \sim(m(y), y, B)$

(ii) a consequentialist if, for all $(m(x), x, A),(m(y), y, B) \in \Omega,[m(x)=m(y), x=$ $y] \Rightarrow(m(x), x, A) \sim(m(y), y, B)$;

(iii) a non-consequentialist if, for some $(m(x), x, A),(m(y), y, B) \in \Omega$, we have $m(x)=m(y)$ but $(m(x), x, A) \succ(m(y), y, B)$. 
Let us begin by providing a simple axiomatic characterization of the two notions of consequentialism. For this purpose, consider the following axioms.

Local Indifference* (LI $\left.^{*}\right)$ : For all $(m(x), x, X),(m(x), x,\{x\}) \in \Omega,(m(x), x, X) \sim$ $(m(x), x,\{x\})$.

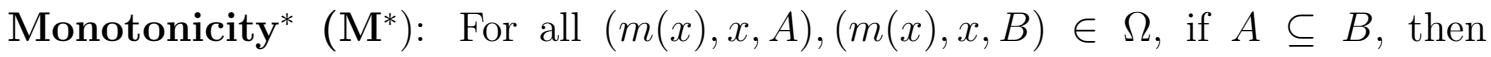
$(m(x), x, B) \succeq(m(x), x, A)$.

Conditional Indifference between No-choice Situations* ${ }^{*}$ CINS $^{*}$ ): For all $(m(x), x,\{x\}),(m(y), y,\{y\}) \in \Omega$, if $m(x)=m(y)$ then $(m(x), x,\{x\}) \sim(m(y)$, $y,\{y\})$.

We may now assert the following:

Theorem 11. $\succeq$ over $\Omega$ satisfies $\left(\mathrm{LI}^{*}\right)$ and $\left(\mathrm{M}^{*}\right)$ if and only if it is consequential.

Proof. It can be verified that, if $\succeq$ is a consequentialist in nature, then it satisfies both $\left(\mathrm{LI}^{*}\right)$ and $\left(\mathrm{M}^{*}\right)$. Suppose now that $\succeq$ satisfies $\left(\mathrm{LI}^{*}\right)$ and $\left(\mathrm{M}^{*}\right)$. We need to show that $\succeq$ must be a consequentialist, that is, for all $(m(x), x, A),(m(y), y, B) \in \Omega$, $[m(x)=m(y), x=y] \Rightarrow(m(x), x, A) \sim(m(y), y, B)$. Let $(m(x), x, A),(m(y), y, B) \in$ $\Omega$ be such that $[m(x)=m(y), x=y]$. By $\left(\mathrm{LI}^{*}\right),(m(x), x,\{x\}) \sim(m(x), x, X)$. Note that $A$ and $B$ must be such that $\{x\} \subseteq A \subseteq X$ and $\{x\} \subseteq B \subseteq X$. By $\left(\mathrm{M}^{*}\right)$, it then follows that $(m(x), x, X) \succeq(m(x), x, A) \succeq(m(x), x,\{x\})$ and $(m(x), x, X) \succeq$ $(m(x), x, B) \succeq(m(x), x,\{x\})$. Noting that $(m(x), x,\{x\}) \sim(m(x), x, X)$, it then follows easily that $(m(x), x,\{x\}) \sim(m(x), x, A)$ and $(m(x), x,\{x\}) \sim(m(x), x, B)$. The transitivity of $\succeq$ now implies that $(m(x), x, A) \sim(m(x), x, B)$.

Theorem 12. $\succeq$ over $\Omega$ satisfies $\left(\mathrm{LI}^{*}\right),\left(\mathrm{M}^{*}\right)$ and $\left(\mathrm{CINS}^{*}\right)$ if and only if it is extremely consequential.

Proof. It can be verified that, if $\succeq$ is an extreme consequentialist in nature, then it satisfies both $\left(\mathrm{LI}^{*}\right),\left(\mathrm{M}^{*}\right)$ and $\left(\mathrm{CINS}^{*}\right)$. Suppose now that $\succeq$ satisfies $\left(\mathrm{LI}^{*}\right),\left(\mathrm{M}^{*}\right)$ and CINS*). We need to show that $\succeq$ is an extreme consequentialist, that is, for all $(m(x), x, A),(m(y), y, B) \in \Omega, m(x)=m(y) \Rightarrow(m(x), x, A) \sim(m(y), y, B)$. Let $\succeq$ over $\Omega$ satisfy $\left(\mathrm{LI}^{*}\right),\left(\mathrm{M}^{*}\right)$ and CINS*), and $(m(x), x, A),(m(y), y, B) \in \Omega$ be such that $m(x)=m(y)$. From Theorem 8, we have $(m(x), x, A) \sim(m(x), x,\{x\})$ and $(m(y), y, B) \sim(m(y), y,\{y\})$. By $\left(\mathrm{CINS}^{*}\right)$ and noting that $m(x)=m(y)$, we have 
$(m(x), x,\{x\}) \sim(m(y), y,\{y\})$. The transitivity of $\succeq$ now implies that $(m(x), x, A) \sim$ $(m(y), y, B)$, as defined.

Turn, now, to the concept of non-consequentialism. Recollect that the experimental studies revealed that there is a situation, where $x \in X$ and $\{x\}$ is a proper subset of $A$ which in turn is a subset of $X$, are such that the Responder's preferences exhibit the following: $(m(x), x,\{x\}) \succ(m(x), x, A)$. This is precisely a situation where the Responder is abhorred by the fact that the Provider has chosen an outrageously unequal method of division $x$, not only from the no-choice situation $\{x\}$, but also from the opportunity set which contains a conspicuously egalitarian method of division. Since our definition of non-consequentialism is so widely embracing that this revealed preference of the Responder can be thereby accommodated. Consider now a subclass of non-consequentialism which reads as follows: the Responder is a fairness-conscious non-consequentialist if, for all $(m(x), x, A),(m(y), y, B) \in \Omega$, if $m(x)=m(y), x=y$ and $[z \geq x$ for all $z \in A \cup B]$, then $|A| \geq|B| \Leftrightarrow(m(x), x, A) \succeq(m(y), y, B)$. This subclass of non-consequentialism may be characterized by introducing the following axioms.

Conditional Simple Preference for Opportunities* (CSPO*): For all $(m(x), x,\{x, y\})$ and all $y \in X$, if $y>x$, then $(m(x), x,\{x, y\}) \succ(m(x), x,\{x\})$.

Conditional Independence* (CIND*): For all $(m(x), x, A),(m(x), x, B) \in \Omega$ and all $z \in X \backslash(A \cup B)$, if $m(x)=m(y)$ then $z \geq x,(m(x), x, A) \succeq(m(x), x, B) \Leftrightarrow$ $(m(x), x, A \cup\{z\}) \succeq(m(x), x, B \cup\{z\})$.

We are now ready to assert the following:

Theorem 13. $\succeq$ over $\Omega$ satisfies (CINS*), (CSPO*) and (CIND*) if and only if it is a fairness-conscious non-consequentialist.

Proof. The proof is similar to that of Theorem 3 and therefore we omit it.

\section{Concluding Remarks}

In view of the undeniable dominance of consequentialism in the whole spectrum of modern welfare economics and social choice theory, it goes without saying that the 
clarification of what we mean by consequentialism and non-consequentialism, what role, if any, consequentialism vis-à-vis non-consequentialism plays in some of the fundamental propositions in normative economics, and what basic axioms, which are mutually exclusive and jointly sufficient to characterize consequentialism and nonconsequentialism, are of fundamental importance. Capitalizing on some recent work including our own, this paper tried to present a coherent account of what we know about these basic questions. In concluding this paper, two qualifying and clarifying remarks are in order.

In the first place, we assumed throughout this paper that the universal set of alternatives, or at least each and every opportunity set which may be presented to the decision-making agent, is a finite set. It is this assumption that allows us to use a simple measure of the richness of opportunities, viz., the number of alternatives in the opportunity set under scrutiny. Needless to say, this is a simplifying assumption which may well be crucially restrictive. This is well known in the related but distinct literature on the measurement of the freedom of choice. Suffice it to note that choosing an outcome $x$ from the singleton set $\{x\}$ may be judged to be inferior to choosing the same outcome $x$ from the larger opportunity set $A$, where $\{x\}$ is a proper subset of $A$, if the decision maker is a non-consequentialist who cares not just about culmination outcomes, but also about opportunity sets which stand behind the choice of culmination outcomes. However, his preference for $(x, A)$ against $(x,\{x\})$ may well be challenged if $A=\{x, y\}$, where $x=$ "a blue car" and $y=$ "an exactly the same car as $x$, except for its color which is only slightly darker than that of $x$ ". In the literature on the freedom of choice, there are several attempts to cope effectively with this problem. We have chosen to stick to the simplest possible treatment in order not to blur the crucial features of our novel problem by being fussy about less than central features such as the measurement of opportunity.

In the second place, there is a well-known alternative to our definition of consequentialism and non-consequentialism. Unlike our definition in terms of the extended preference ordering over the pairs of culmination outcomes and background opportunity sets, this alternative definition makes use of the extended preference ordering defined over the pairs of culmination outcomes and social decision-making procedures through which these outcomes are brought about. Due recognition of the importance of procedural considerations vis-à-vis consequential considerations abound in the literature. Suffice it to refer to Schumpeter (1942), Arrow (1951) and Lindbeck (1988) 
as a small sample list of economists who, in their respective ways, recognized the need for including social decision-making procedures or mechanisms within the extended evaluative framework of normative economics. This extended framework provides us with an alternative method to articulate consequentialism and non-consequentialism. See, for example, Hansson $(1992 ; 1996)$ who explored the possibility of resolving Arrow's impossibility result in the extended framework, and Gaertner and Xu (2004) who investigated the effects of procedures on decision maker's choices.

The gate is wide open for further exploration of normative economics which goes beyond the traditional confinement of welfarist-consequentialism. 


\section{References}

Arrow, K. J. (1951). Social Choice and Individual Values, New York: Wiley. Second ed., 1963.

Bolton, G.E., Brandts, J., and A. Ockenfels. "Fair Procedures: Evidence from Games Involving Lotteries," Economic Journal 117, pp.1054-1076.

Bossert, W., Pattanaik, P. K. and Y. Xu (1994). "Ranking Opportunity Set: An Axiomatic Approach," Journal of Economic Theory 63, pp.326-345.

Bossert, W., Pattanaik, P. K. and Y. Xu (2003). "Similarity of Options and the Measurement of Diversity," Journal of Theoretical Politics 15, pp.405-421.

Broome, J. (2003). "Representing an Ordering When the Population Varies," Social Choice and Welfare 20, pp.243-246.

Cox, J. C., D. Friedman and S. Gjerstad (2006). "A Tractable Model of Reciprocity and Fairness," Games and Economic Behavior, forthcoming.

Cox, J. C., D. Friedman and V. Sadiraj (2005). "Revealed Altruism," Working Paper, Department of Economics, University of Arizona.

Dworkin, G. (1982). "Is More Choice Better Than Less?" in French, P. A., Uehling, Jr. T. E. and H. K. Wettstein, eds., Midwest Studies in Philosophy, VII, Social and Political Philosophy, Minneapolis: University of Minnesota Press, pp.47-61.

Frey, B. S. and A. Stutzer (2004). "Beyond Outcomes: Measuring Procedural Utility," Oxford Economic Papers 57, pp.90-111.

Gaertner, W. and Y. Xu (2004). "Procedural Choice," Economic Theory 24, pp. 335-349.

Gravel, N. (1994). "Can a Ranking of Opportunity Sets Attach an Intrinsic Importance to Freedom of Choice?" American Economic Review: Papers and Proceedings 84, pp.454-458.

Gravel, N. (1998). "Ranking Opportunity Sets on the Basis of Their Freedom of Choice and Their Ability to Satisfy Preferences: A Difficulty," Social Choice and Welfare 15, pp.371-382. 
Hansson, S. O. (1992). "A Procedural Model of Voting," Theory and Decision 32, pp.269-301.

Hansson, S. O. (1996). "Social Choice with Procedural Preferences," Social Choice and Welfare 13, pp.215-230.

Iwata, Y. (2006). "Consequences, Opportunities, and Arrovian Theorems with Consequentialist Domains," paper presented at the Spring Meeting of the Japanese Economic Association.

Jones, P. and R. Sugden (1982). "Evaluating Choices," International Review of Law and Economics 2, pp.47-65.

Lindbeck, A. (1988). "Individual Welfare and Welfare State Policy," European Economic Review 32, pp.295-318.

Pattanaik, P. K. and K. Suzumura (1994). "Rights, Welfarism and Social Choice," American Economic Review: Papers and Proceedings 84, pp.435-439.

Pattanaik, P. K. and K. Suzumura (1996). "Individual Rights and Social Evaluation," Oxford Economic Papers 48, pp.194-212.

Pattanaik, P. K. and Y. Xu (1990). "On Ranking Opportunity Sets in Terms of Freedom of Choice," Recherches Economiques de Louvain 56, pp.383-390.

Pattanaik, P. K. and Y. Xu (2000). "On Diversity and Freedom of Choice," Mathematical Social Sciences 40, pp.123-130.

Pattanaik, P. K. and Y. Xu (2006). "Ordinal Distance, Dominance, and the Measurement of Diversity," Working Paper 06-57, Andrew Young School of Policy Studies, Georgia State University.

Rabin, M. (1993). "Incorporating Fairness into Game Theory and Economics," American Economic Review 83, pp.1281-1302.

Rabin, M. (2002). "A Perspective on Psychology and Economics," European Economic Review 46, pp.657-685.

Schumpeter, J. A. (1942). Capitalism, Socialism, and Democracy, New York: Harper \& Brothers. 
Sen, A. K. (1985). Commodities and Capabilities. Amsterdam: North-Holland.

Sen, A. K. (1988). "Freedom of Choice: Concept and Content," European Economic Review 32, pp.269-294.

Sen, A. K. (1993). "Markets and Freedoms: Achievements and Limitations of the Market Mechanism in Promoting Individual Freedom," Oxford Economic Papers 45, pp.519-541.

Sen, A. K. (1995). "Rationality and Social Choice," American Economic Review 85, pp.1-24.

Sen, A. K. (1996). "Maximization and the Act of Choice," Econometrica 65, pp.745779 .

Simon, H.A. (1976). "From Substantive to Procedural Rationality," in S.J. Latsis (ed.) Methods and Appraisal in Economics. Cambridge, MA.: Cambridge University Press.

Simon, H.A. (1978). "Rationality as a Process and Product of Thought," American Economic Review 68, pp.1-16.

Suzumura, K. (1999). "Consequences, Opportunities, and Procedures," Social Choice and Welfare 16, pp.17-40.

Suzumura, K. (2000). "Welfare Economics Beyond Welfarist-Consequentialism," Japanese Economic Review 51, pp.1-32.

Suzumura, K. and Y. Xu (2001). "Characterizations of Consequentialism and Non-consequentialism," Journal of Economic Theory 101, pp.423-436.

Suzumura, K. and Y. Xu (2003). "Consequences, Opportunities, and Generalized Consequentialism and Non-consequentialism," Journal of Economic Theory 111, pp.293-304.

Suzumura, K. and Y. Xu (2004). "Welfarist-Consequentialism, Similarity of Attitudes, and Arrow's General Impossibility Theorem," Social Choice and Welfare 22, pp.237-251 . 